\title{
Adding Wavelength Shifter Molecules to the Sudbury Neutrino Observatory
}

\author{
by \\ Etienne Rollin, B.Sc. \\ A thesis submitted to \\ the Faculty of Graduate Studies and Research \\ in partial fulfillment of \\ the requirements for the degree of \\ Master of Science \\ Ottawa-Carleton Institute for Physics \\ Department of Physics \\ Carleton University
Ottawa, Ontario, Canada \\ January 2005 \\ (C) copyright \\ 2005, Etienne Rollin
}




$\begin{array}{ll}\begin{array}{l}\text { Library and } \\ \text { Archives Canada }\end{array} & \begin{array}{l}\text { Bibliothèque et } \\ \text { Archives Canada }\end{array} \\ \begin{array}{l}\text { Published Heritage } \\ \text { Branch }\end{array} & \begin{array}{l}\text { Direction du } \\ \text { Patrimoine de l'édition }\end{array} \\ \begin{array}{l}\text { 395 Wellington Street } \\ \text { Ottawa ON K1A ON4 }\end{array} & \begin{array}{l}\text { 395, rue Wellington } \\ \text { Ottawa ON K1A ON4 } \\ \text { Canada }\end{array}\end{array}$

Your file Votre référence

ISBN: 0-494-00789-3

Ourfile Notre référence

ISBN: 0-494-00789-3

NOTICE:

The author has granted a nonexclusive license allowing Library and Archives Canada to reproduce, publish, archive, preserve, conserve, communicate to the public by telecommunication or on the Internet, loan, distribute and sell theses worldwide, for commercial or noncommercial purposes, in microform, paper, electronic and/or any other formats.

The author retains copyright ownership and moral rights in this thesis. Neither the thesis nor substantial extracts from it may be printed or otherwise reproduced without the author's permission.
AVIS:

L'auteur a accordé une licence non exclusive permettant à la Bibliothèque et Archives Canada de reproduire, publier, archiver, sauvegarder, conserver, transmettre au public par télécommunication ou par l'Internet, prêter, distribuer et vendre des thèses partout dans le monde, à des fins commerciales ou autres, sur support microforme, papier, électronique et/ou autres formats.

L'auteur conserve la propriété du droit d'auteur et des droits moraux qui protège cette thèse. $\mathrm{Ni}$ la thèse ni des extraits substantiels de celle-ci ne doivent être imprimés ou autrement reproduits sans son autorisation.
In compliance with the Canadian

Privacy Act some supporting forms may have been removed from this thesis.

While these forms may be included in the document page count, their removal does not represent any loss of content from the thesis.
Conformément à la loi canadienne sur la protection de la vie privée, quelques formulaires secondaires ont été enlevés de cette thèse.

Bien que ces formulaires aient inclus dans la pagination, il n'y aura aucun contenu manquant.

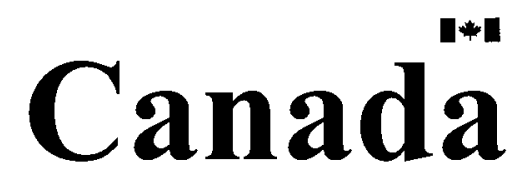




\begin{abstract}
This thesis provides a detailed study of the benefits of adding wavelength shifter molecules (WLS) to the heavy water of the Sudbury Neutrino Observatory (SNO). Their absorption and re-emission spectra allow the conversion of ultraviolet Cerenkov photons into longer wavelength photons, that would be more easily detected. Measurements of the optical properties of five candidates show that only two of them, Alexa Fluor and Carbostyril, are suitable.

The analysis indicates that WLS would increase the number of detected photons, lead to more uniform distributions for the reconstructed position and energy, allow a larger fiducial volume and a lower energy threshold for the study of solar neutrinos. The goal is to determine whether adding WLS to SNO will allow a direct measurement of matter induced neutrino oscillations. Finally, a cosmic ray telescope has been developed and shows promising results for an independent measurement of the optical properties of WLS.
\end{abstract}

iii 
À Maman, Papa et Katia. Soyez aussi fiers de vous que vous l'êtes de moi car vos encouragements furent nécessaires pour me rendre aussi loin. Et ce n'est pas fini! Merci beaucoup. 


\section{Acknowledgments}

There are too many people to thank, but here are those who significantly helped me, both morally and physically, in completing the work depicted in this thesis. Many thanks to my mother and father, Cécile and Rémi, my girlfriend Katia and my family for the encouragements and support during my graduate studies. I am also grateful to Alain Bellerive and Cliff Hargrove for the technical help, but also for their priceless advice. Many thanks to, in alphabetical order, Xin Dai, Ranpal Dosanjh, Pierre-Luc Drouin, Pascal Elahi, Rachel Faust, Darren Grant, Philippe Gravelle, Louise Heelan, Richard Hemingway, Christiane Mallett, Cathy Mifflin, Olivier Simard, Gordana Tesic, David Waller, the physics faculty members of Carleton University and the SNO collaboration for having given me a chance to learn about particle physics and the dynamics of a large collaboration, without whom this thesis would not have been possible. Finally, special thanks to Rachel Faust and David Waller who compensated for my deficient English by helping me in correcting this thesis. 


\section{Statement of Originality}

I certify that this thesis is the product of my own work, and that any ideas or quotations from the work of other people are fully acknowledged in accordance with the standard referencing practices of the discipline. I acknowledge the helpful guidance and support of my supervisor, Professor Alain Bellerive, and also Professor Emeritus Cliff Hargrove who designed the WLS telescope.

As a part of a large collaboration, I had to use tools developed by other people. I used the SNOMAN program and the QPhysics package created by the SNO collaboration, and ROOT, a CERN analysis package. I want to acknowledge the work of Pascal Elahi who added the photon interaction with WLS molecules in SNOMAN.

Measurements of the optical properties listed in Chapter 2 of my thesis were done by Xin Dai, Cathy Mifflin and Christiane Mallett, chemists trained for this type of work.

A stand-alone Monte Carlo program for the WLS telescope was written by myself with help from Prof. Hargrove who proposed a routine for the WLS effect on photons. With advice, I performed the Monte Carlo simulations, developed 
advanced data analysis routines and summarized the implications of their results. I should also recognize the work of two summer students, Alex Davis and Rachel Faust who worked on building and calibrating the WLS telescope.

While working on my Master degree's at Carleton University with the SNO collaboration, I signed three refereed articles:

1. Measurement of the Total Active ${ }^{8} B$ Solar Neutrino Flux at the Sudbury Neutrino Observatory with Enhanced Neutral Current Sensitivity, S. N. Ahmed et al., Phys. Rev. Lett. 92, 181301 (2004).

2. Constraints on Nucleon Decay via Invisible Modes from the Sudbury Neutrino Observatory, S. N. Ahmed et al., Phys. Rev. Lett. 92, 102004 (2004)

3. Electron Antineutrino Search at the Sudbury Neutrino Observatory, S. N. Ahmed et al., Phys. Rev. D70, 093014 (2004).

I also produced two internal notes for the SNO collaboration and gave many talks, one of which was at the Canadian Association of Physicists (CAP) congress in Winnipeg in June 2004. 


\section{Contents}

Abstract ...............................

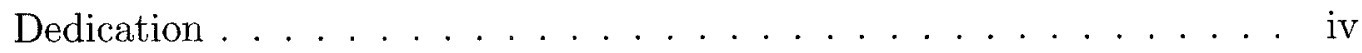

Acknowledgments ..................... . . .

Statement of Originality .................. vi

List of Tables

List of Figures $\quad$ xii

1 Introduction $\quad 1$

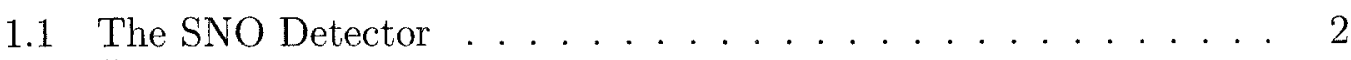

1.2 Čerenkov Radiation . . . . . . . . . . . . . . . . . . 4

2 Properties of Wavelength Shifters $\quad 6$

2.1 Selection Criteria . . . . . . . . . . . . . 8

2.2 Candidate Selection . . . . . . . . . . . . . . . . . . . . . . . . . . . . . . . 9

2.2.1 Solubility and Removal . . . . . . . . . . . . . . . 9

2.2 .2 Molecule Stability . . . . . . . . . . . . . . . 10

2.2.3 Absorption and Re-emission Spectra . . . . . . . . . . 10

2.2.4 Quantum Efficiency ... . . . . . . . . . . . . 13

2.2 .5 Re-emission Time . . . . . . . . . . . . . . 13

2.3 Summary . . . . . . . . . . . . . . . . . 16

3 Monte Carlo Simulations for SNO $\quad 17$

3.1 Parameters in SNOMAN . . . . . . . . . . . . . 17

3.2 Determination of the WLS Concentration . . . . . . . . . . . 18

3.3 Gain of Light . . . . . . . . . . . . . . . . . . . . . . . . 20

3.3.1 Analytical Approximation . . . . . . . . . . . 20

3.3.2 Monte Carlo Dataset . . . . . . . . . . . . . . . . 20

viii 
3.3.3 Energy Resolution Uncertainty . . . . . . . . . . . . . . 21

3.3.4 PMT Time distribution ... . . . . . . . . . . . . 22

3.4 Significance of the Signal over the Background . . . . . . . . . . 22

3.5 Isotropy of the WLS Light . . . . . . . . . . . . . . . . . . . 24

3.5 .1 Uniformity . . . . . . . . . . . . . . 27

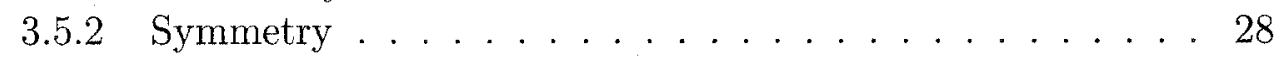

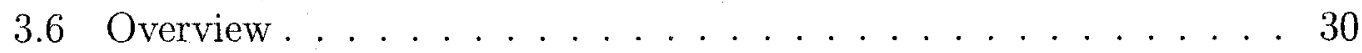

4 Neutrino Energy Spectrum $\quad 32$

4.1 Neutrino Oscillation Formalism . . . . . . . . . . . . . . 33

4.2 Test Statistic . . . . . . . . . . . . . . . . . . . . . . . . . . . . . . . . . .

4.3 Spectral Distortion Analysis . . . . . . . . . . . . . 36

4.3.1 5.5 MeV Energy Threshold . . . . . . . . . . . . . . 37

4.3.2 $3.7 \mathrm{MeV}$ Energy Threshold . . . . . . . . . . . . . . . 39

$4.3 .3 \quad 3.25 \mathrm{MeV}$ Energy Threshold . . . . . . . . . . . . . . . 41

4.4 Summary ... . . . . . . . . . . . . . . 43

5 Wavelength Shifter Cosmic Ray Telescope 46

5.1 Description of the Apparatus . . . . . . . . . . . . . . 47

5.1 .1 Čerenkov Cells . . . . . . . . . . . . . . . 47

5.1 .2 Scintillators . . . . . . . . . . . . . . . . 50

5.1 .3 Data Acquisition System . . . . . . . . . . . . . . 51

5.2 Monte Carlo Simulation . . . . . . . . . . . . . . . 51

5.3 Geometric Acceptance . . . . . . . . . . . . . . . 52

5.3 .1 Improvements . . . . . . . . . . . . . . . . 53

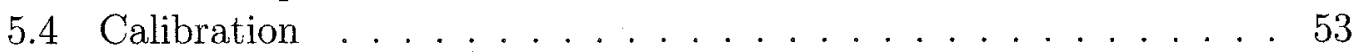

5.4 Single-Photon Calibration . . . . . . . . . . . 53

5.4 .2 Multi-Photon Calibration ............ 54

5.5 Timing and Gain Measurements . . . . . . . . . . . . . . . . 56

5.5 .1 Time Measurement . . . . . . . . . . . . . 58

5.5 .2 Gain Measurement . . . . . . . . . . . . 60

5.6 Summary . . . . . . . . . . . . . . . . . 63

6 Conclusion $\quad 64$

$\begin{array}{ll}\text { Bibliography } & 66\end{array}$

$\begin{array}{ll}\text { A WLS Candidate Structures } & 67\end{array}$ 
B Analytic Approximation 68

B.1 Čerenkov Radiation . . . . . . . . . . . . . . 68

B.2 WLS Light. . . . . . . . . . . . . . . . 68 


\section{List of Tables}

2.1 Quantum efficiency, re-emission peak position, re-emission time and price of the Alexa Fluor and Carbostyril . . . . . . . 16

3.1 Number of detected photons as a function of the concentration of WLS. . . . . . . . . . . . . . . . . . 20

3.2 Energy threshold that maximize the statistical significance of the signal. . . . . . . . . . . . . . . . . . 27

5.1 Geometric acceptance of the WLS telescope for Cerenkov and WLS radiations. . . . . . . . . . . . . . . . . . . 53

5.2 FWHM of the timing distributions for different configurations of the WLS telescope. . . . . . . . . . . . . . . . . . . . 60

5.3 Mean amplitude of pulses for different configuration of the WLS telescope. . . . . . . . . . . . . . . . . 63 


\section{List of Figures}

1.1 Simplified view of the SNO detector. . . . . . . . . . . 5

2.1 Normalized response of the detector as a function of the wavelength superimposed on the Cerenkov spectrum. . . . . . . . 7

2.2 Absorption an re-emission spectra as a function of the wavelength for the Alexa Fluor $350 . \ldots \ldots \ldots$. . . . . . . . . . 11

2.3 Absorption an re-emission spectra as a function of the wavelength for the Carbostyril 124. . . . . . . . . . . . . . . . 12

2.4 Remission time spectrum for the Alexa Fluor 350. . . . . . . . 14

2.5 Remission time spectrum for the Carbostyril 124. . . . . . . 15

3.1 Nhits distribution as a function of the concentration of the WLS solution for $10 \mathrm{MeV}$ electrons. . . . . . . . . . . . . . 19

3.2 Gain of light as a function of the concentration of the WLS solution. 21

3.3 PMT time distribution as a function of the concentration of the WLS solution. . . . . . . . . . . . . . . . . 23

3.4 Signal energy cut over background without WLS. . . . . . . 25

3.5 Signal energy cut over background with WLS. . . . . . . . 26

3.6 Uniformity of the signal after reconstruction. . . . . . . . . . 29

3.7 Symmetry of the signal with the NCD . . . . . . . . 31

4.1 Reconstructed kinetic energy of CC events for a simulated SNO $\mathrm{D}_{2} \mathrm{O}+$ Salt dataset. . . . . . . . . . . . . 35

4.2 MSW plot for the current SNO configuration. . . . . . . . . 40

4.3 Number of years before excluding undistorted spectrum hypothesis for a $3.7 \mathrm{MeV}$ threshold. . . . . . . . . . . . . . . . . 42

4.4 Number of years before excluding undistorted spectrum hypothesis for the best case scenario. . . . . . . . . . . . . . . . 44 
5.1 Design of the WLS telescope. . . . . . . . . . . . . 48

5.2 Different configurations for data taking of the WLS telescope. . . 49

5.3 Logic circuitry of the WLS telescope. . . . . . . . . . . 50

5.4 Example of a single-photon peak spectrum. . . . . . . . . . . 54

5.5 One-photon peak position as a function of the applied voltage on the PMTs. . . . . . . . . . . . . . . . . 55

5.6 Multi-photon mean pulse amplitude as a function of the applied voltage on the PMT. . . . . . . . . . . . 57

5.7 Hypothetical situation for the simple model used to determine the re-emission time from the pulse widths. . . . . . . . . . . . 59

5.8 FWHM of two Gaussians as a function of the difference of the means. . . . . . . . . . . . . . . . . . . 6 61 


\section{Chapter 1}

\section{Introduction}

The Standard Model of elementary particles asserts that the material in the universe is made up of fermions interacting through fields to which we associate particles called bosons. Fermions are from two types, quarks and leptons, that can be classified by generations:

$$
\left(\begin{array}{l}
u \\
d
\end{array}\right)\left(\begin{array}{l}
c \\
s
\end{array}\right)\left(\begin{array}{l}
t \\
b
\end{array}\right)
$$

for the quarks and

$$
\left(\begin{array}{l}
e \\
\nu_{e}
\end{array}\right)\left(\begin{array}{l}
\mu \\
\nu_{\mu}
\end{array}\right)\left(\begin{array}{l}
\tau \\
\nu_{\tau}
\end{array}\right)
$$

for the leptons.

For more than thirty years, particle astrophysicists were puzzled by the low flux of solar electron neutrinos $\left(\nu_{e}\right)$ that reach the Earth. In 2002, the Sudbury Neutrino Observatory (SNO) solved this solar neutrino problem by measuring both the $\nu_{e}$ and the total active neutrino fluxes $\left(\nu_{e}, \nu_{\mu}, \nu_{\tau}\right)$, providing strong evidence for solar neutrino flavour transformation [1]. 
Neutrino experiments now aim to do a precise measurement of the oscillation parameters that define the behavior of neutrino transformation in vacuum and matter. In order to continue exploring its unique methods of measuring neutrino fluxes, the SNO collaboration studied the possibility of enhancing the detector by using wavelength shifting molecules (WLS). The WLS would have the effect of shifting the wavelength of Cerenkov photons from the ultraviolet (UV) part to the visible range of the spectrum.

\subsection{The SNO Detector}

SNO is a one thousand tons heavy water $\left(\mathrm{D}_{2} \mathrm{O}\right)$ detector buried two kilometers underground in Sudbury, Canada, built with the objective of measuring the total solar neutrino flux. Its main component is an acrylic vessel (AV) that contains the $\mathrm{D}_{2} \mathrm{O}$, and is surrounded by 9456 photomultiplier tubes (PMTs) that detect the Čerenkov light emitted by relativistic charged particles. The apparatus is submerged in regular ultra-pure water to support the AV and to shield the $\mathrm{D}_{2} \mathrm{O}$ from natural radioactivity in the cavity. Figure 1.1 shows a simplified view of the detector. SNO can detect three types of interactions. Each interaction has a sensitivity to the three types of neutrino flavours. The charged current (CC) and the neutral current (NC) interactions are the most important and the most common. The third type of interaction is the electron scattering (ES) that happens less frequently. The following equations summarize all three types of 
signal interactions:

$$
\begin{array}{cc}
\nu_{e}+d \rightarrow e^{-}+p+p & (C C) \\
\nu_{x}+d \rightarrow p+n+\nu_{x} & (N C) \\
e^{-}+\nu_{x} \rightarrow e^{-}+\nu_{x} & (E S) .
\end{array}
$$

The flux $(\phi)$ for each interaction is related to the neutrino flux by these equations:

$$
\begin{aligned}
\phi_{C C} & =\phi\left(\nu_{e}\right) \\
\phi_{N C} & =\phi\left(\nu_{e}\right)+\phi\left(\nu_{\mu, \tau}\right) \\
\phi_{E S} & =\phi\left(\nu_{e}\right)+0.16 \times \phi\left(\nu_{\mu, \tau}\right) .
\end{aligned}
$$

The CC interaction is sensitive to $\nu_{e}$ only, while the $\mathrm{NC}$ interaction is equally sensitive to all three neutrino flavours. Therefore, the ratio $\mathrm{CC} / \mathrm{NC}$ offers the possibility of distinguishing the $\nu_{e}$ and $\nu_{\mu, \tau}$ components of the solar neutrino flux [2]. Based on the proportion of each flavour as a function of the neutrino energy, the SNO collaboration has been able to measure the neutrino oscillation parameters $\delta m^{2}$ and $\tan ^{2} \theta$. The parameter $\delta m^{2}$ is the square of the mass difference between two of the neutrino mass eigenstates and $\theta$ is the mixing angle.

The SNO detector has been enhanced twice to increase its sensitivity. The NC interaction produces a free neutron. Since the cross-section of neutron capture on deuteron or oxygen is relatively low, two tonnes of ultra pure $\mathrm{NaCl}$ have been added to the $\mathrm{D}_{2} \mathrm{O}$ to increase the neutron capture efficiency. Chlorine had the effect of increasing the detection efficiency of neutron by a factor of three. Unfortunately, even after the addition of the salt, there was a large correlation between the NC and CC signals, so it was decided to replace the salt with neutral current detectors (NCDs). The NCDs are metallic tubes filled with ${ }^{3} \mathrm{He}$ which 
act as proportional counters for the detection of neutrons. With NCDs, NC signal will be discriminated on an event-by-event basis. A fourth phase of the experiment could be the addition of WLS to the $\mathrm{D}_{2} \mathrm{O}$. Adding WLS to the $\mathrm{D}_{2} \mathrm{O}$ of the SNO detector would significantly increase the number of photons detected from inside the AV. In addition to the increase in the number of detected photons, it could be used in conjunction with other devices like NCDs.

\section{2 Čerenkov Radiation}

SNO detects neutrinos indirectly from particles produced by the three interactions listed in Equations 1.1 to 1.3. Each interaction produces, directly or indirectly, one or more relativistic electrons from which Čerenkov photons radiate if the electrons's velocity is greater than the speed of light in the medium of propagation. The radiation is emitted as a coherent wavefront with a constant angle with respect to the direction of propagation. This results in a cone of light.

In the SNO detector, this light is detected by the PMTs and the Cerenkov cone projected on them forms an ellipse, from which the position, the direction and the energy of the electrons can be extracted. 


\section{SNO Detector}

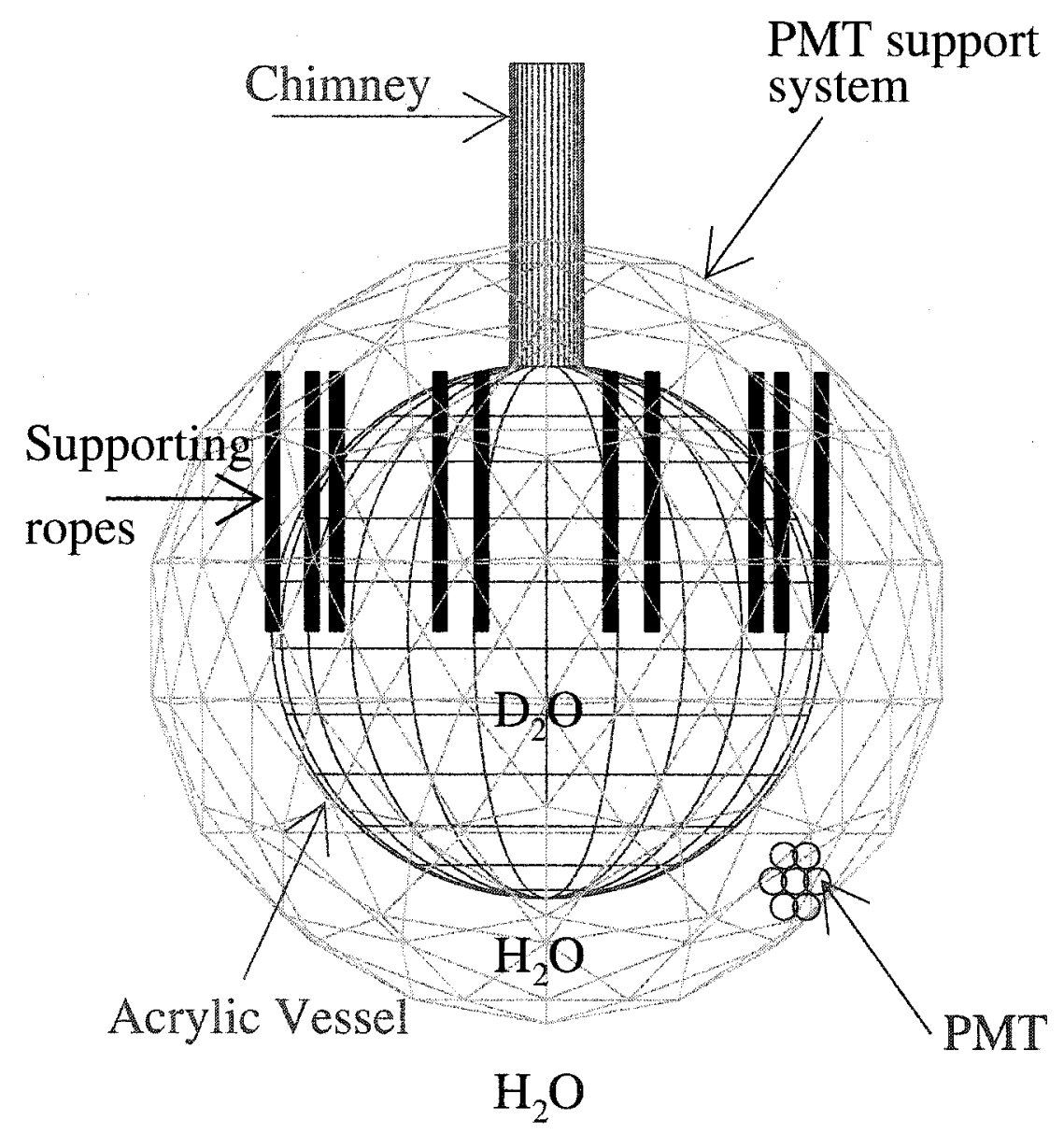

Figure 1.1: Simplified view of the SNO detector. The acrylic vessel and its chimney are in blue, the PMT support system in gray, the support cables for the AV in black and the PMTs in red covering all over the PMT support system. The AV contains the $\mathrm{D}_{2} \mathrm{O}$ while the outer volume is filled with $\mathrm{H}_{2} \mathrm{O}$. The $\mathrm{AV}$ has a diameter of 12 meters. View this plot in color. 


\section{Chapter 2}

\section{Properties of Wavelength}

\section{Shifters}

A WLS is defined as a molecule that absorbs photons and re-emits them at a longer wavelength by fluorescence. Previous studies showed that adding WLS to Cerenkov detectors increases the detected amount of light by a factor between two and three $[3,4,5]$. Results should be similar in SNO; an increase in the number of detected photons per event is expected when adding WLS to the $\mathrm{D}_{2} \mathrm{O}$. Due to the geometry of the detector, every Cerenkov photon has to travel through at least $5.5 \mathrm{~cm}$ of acrylic before being detected by one of the 9456 PMTs (see Figure 1.1). Transmission of the SNO acrylic and the PMT quantum efficiency as a function of the wavelength, together with the Cerenkov spectrum generated by relativistic charged particles, in Figure 2.1. Therefore, most of the UV photons are absorbed by the acrylic and the detector response is very poor below $300 \mathrm{~nm}$. Consequently, an ideal WLS candidate for SNO should change the wavelength of Cerenkov photons from below $300 \mathrm{~nm}$ to $400 \mathrm{~nm}$, the range where the detector's response is optimal as shown in Figure 2.1. Not shown 


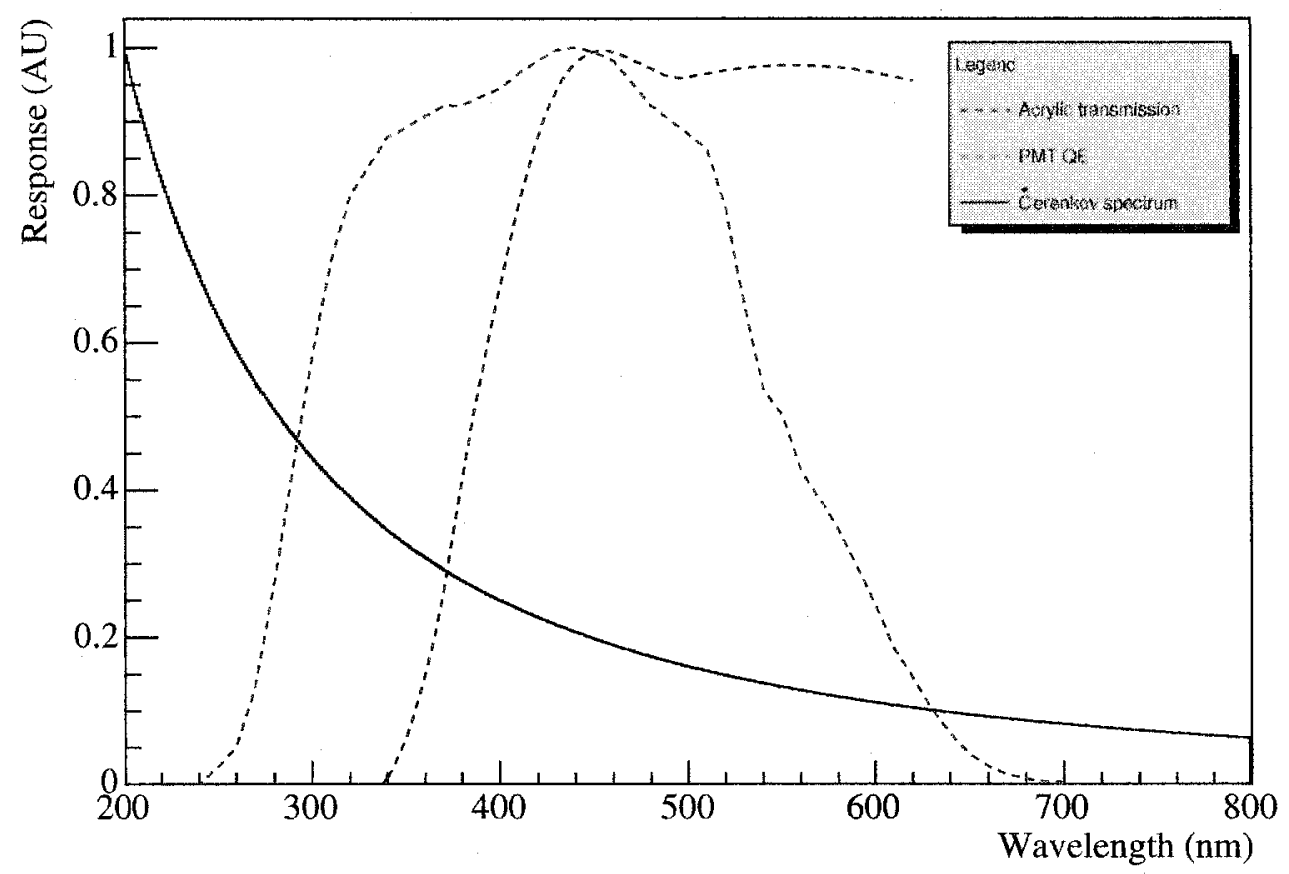

Figure 2.1: Transmission of the acrylic (dashed blue) and PMT QE (dashed red) as a function of wavelength superimposed on the Cerenkov spectrum in solid black. Note that all three distributions are normalized to have unity at the maximum of the distributions. View this plot in color.

on the plot are the $\mathrm{D}_{2} \mathrm{O}$ and $\mathrm{H}_{2} \mathrm{O}$ transmission curves, since the response of the detector is driven mainly by the acrylic at short wavelength and the quantum efficiency (QE) of the PMTs at long wavelength.

The possibility of boosting the Cerenkov signal from neutrino events with the addition of WLS in the $\mathrm{D}_{2} \mathrm{O}$, combined with the fact that background photons from outside the $\mathrm{D}_{2} \mathrm{O}$ volume are expected to remain at a a very low level, should result in a significant increase in the detector's efficiency, as it will be discussed in Chapter 3. 
Since the light re-emitted by the WLS is isotropic, the CC and NC signals would be difficult to separate without the directional information from the Cerenkov radiation. To understand this process, Monte Carlo simulations would be necessary to find out if the distributions of photons cone would be significantly different in order to maintain a low uncertainty on the fitted event position.

\subsection{Selection Criteria}

A list of desirable WLS characteristics are as follows:

1. soluble in and removable from water;

2. stable and long solution lifetime;

3. no adverse interaction with the materials used in SNO's underground water system, including acrylic, polypropylene, $\mathrm{MnOx}$ beads and $\mathrm{HTiO}$ absorbent;

4. high photon absorption below $380 \mathrm{~nm}$ and emission around $400-500 \mathrm{~nm}$;

5. no significant overlap between excitation and emission regions;

6. low $\mathrm{pH}$-dependence in the neutral $\mathrm{pH}$ range;

7. high quantum efficiency (i.e. re-emission probability);

8. no more than a few nanoseconds of re-emission time.

Each criterion is reviewed in detail in the following sections of this chapter. 


\subsection{Candidate Selection}

Five WLS compounds were preselected from many candidates: 7-hydroxy-4methylcoumarin, 7-hydroxy-4-methylcoumarin-3-acetic acid, 7-hydroxycoumarin3-carboxylic acid, Alexa Fluor 350 and Carbostyril 124 (see Appendix A for chemical structures). Some of these were chosen for their standard use in industries, while others were carefully picked for their suitability given the list of requirements. Tests were performed on the five candidates to quantify their absorption coefficients, fluorescence decay time, quantum efficiency, interactions with different media and removal rate using different techniques. Most tests were performed more than twice to ensure reproducibility.

\subsubsection{Solubility and Removal}

To avoid any modification to the detector, the WLS additive ought to be soluble in $\mathrm{D}_{2} \mathrm{O}$. A concentration between 0.01 and $100 \mathrm{ppm}$ should be easily obtained without having to add a buffer, which would alter the $\mathrm{pH}$ of the solution or introduce unnecessary material. Unfortunately, all three 7-hydroxy candidates showed a pH dependence, meaning that those molecules were efficient in converting UV photons at a $\mathrm{pH}$ much higher than the one of the $\mathrm{D}_{2} \mathrm{O}[6]$.

Since the $\mathrm{D}_{2} \mathrm{O}$ has been loaned by Atomic Energy of Canada Limited (AECL), it should be returned additive-free at the end of the SNO experiment. Removal techniques were therefore studied. Because WLS compounds are generally organic, activated charcoal is efficient at removing WLS from water [6]. A second technique that uses biobeads also extracted WLS, but at a much lower rate. Therefore, having two efficient techniques available, removal of WLS should not be an issue in the selection of a candidate. 


\subsubsection{Molecule Stability}

WLS were tested for changes in their optical properties over a period of two months. Unfortunately, a variation in the emission spectrum of the 7-hydroxy-4methylcoumarin-3-acetic acid was observed within those two months, a lifetime much lower than what is required for the SNO experiment.

No significant change in the amplitude of the excitation peak was found when the other WLS were placed in contact with acrylic for a period of six months; no accumulation of WLS was observed. Similarly, tests were done using other materials that are in contact with the $\mathrm{D}_{2} \mathrm{O}$ in the detector. The 7-hydroxy-4methylcoumarin and 7-hydroxycoumarin-3-carboxylic acid seemed to react with the HTiO absorbent, which was unacceptable. The last two candidates, Alexa Fluor 350 and Carbostyril 124, did not interact with the HTiO absorbent nor with the $\mathrm{MnOx}$ beads. Since they both succeeded at all tests, studies were focused on their properties as described in the following sections.

\subsubsection{Absorption and Re-emission Spectra}

Absorption and re-emission spectra are the most important features for the evaluation of the performance of a WLS. A strong absorption is necessary because it results in a shorter distance between the generation and excitation positions, and facilitates the reconstruction of the event afterward. In addition, the re-emission spectrum should be sharp and close to the wavelength that the detector is most sensitive to, which is about $400 \mathrm{~nm}$ in the case of SNO. Finally, absorption and re-emission spectra should not overlap otherwise multiple excitation and emission cycles could occur. This would spread the time distribution needed to fit the event position since each cycle lasts about 6 ns. A quick 


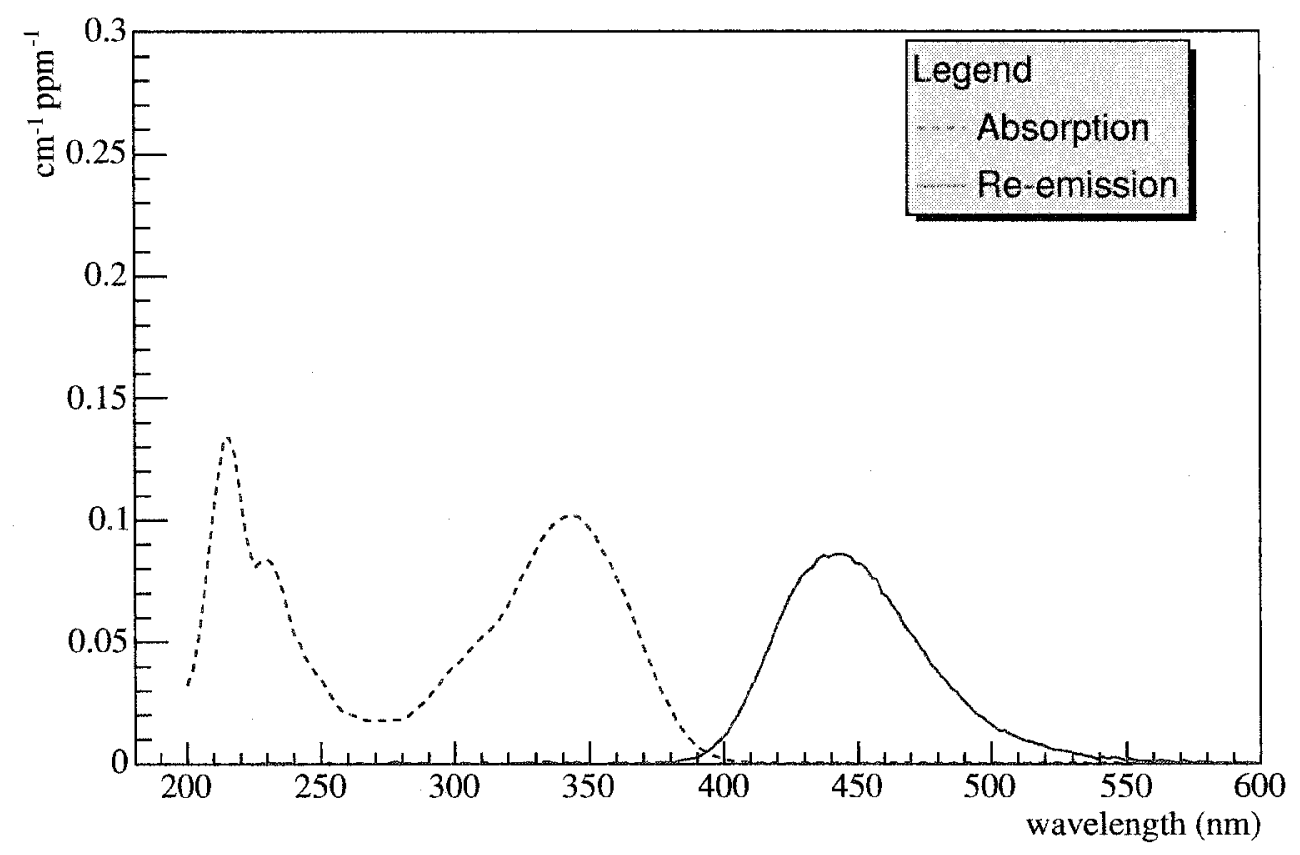

Figure 2.2: Absorption (dotted red) and re-emission (solid blue) spectra as a function of wavelength for the Alexa Fluor 350 . 


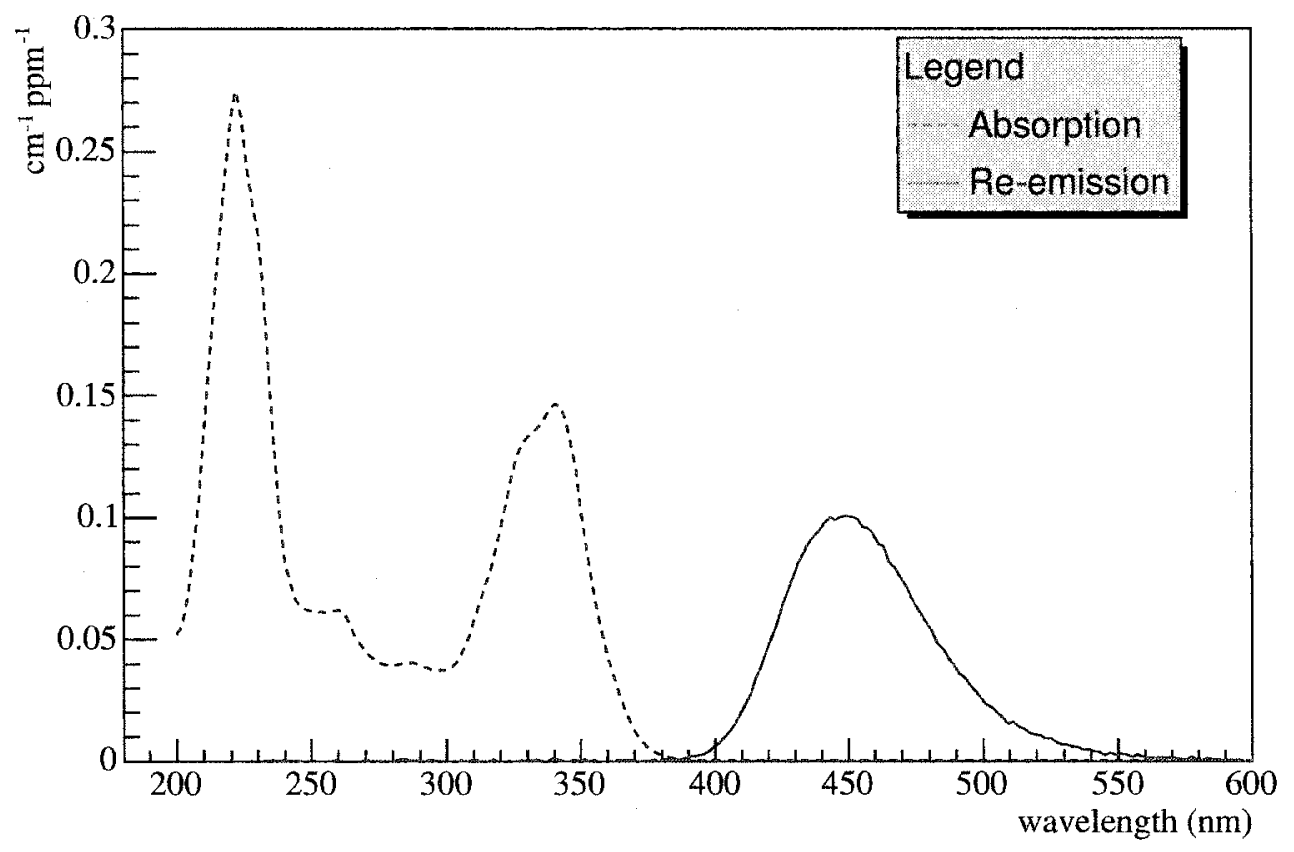

Figure 2.3: Absorption (dotted red) and re-emission (solid blue) spectra as a function of wavelength for the Carbostyril 124 . 
absorption lowers the uncertainty in the position.

Two Carleton University chemists, Xin Dai and Cathy Mifflin, measured the absorption and re-emission spectra with a Cary Eclipse Fluorescence Spectrophotometer made by Varian. Results are plotted in Figures 2.2 and 2.3 and show similar spectra for both candidates. The most important difference is the longer tail in the second re-emission peak of the Alexa Fluor. The presence of absorption at a wavelength higher than $400 \mathrm{~nm}$ could interfere with detected Cerenkov light and would decrease the resolution of the SNO detector.

\subsubsection{Quantum Efficiency}

Quantum efficiency $(\mathrm{QE})$ is a simpler way to represent absorption and reemission spectra. It is the probability of re-emission of a photon absorbed by a WLS molecule. The higher the QE, the greater the number of detectable photons. As shown in Table 2.1, the QE for both candidates is very high: 93\% for the Alexa Fluor and $98 \%$ for the Carbostyril. Therefore, QE has a minimal influence on the choice of the best candidate.

\subsubsection{Re-emission Time}

As discussed previously, the re-emission time influences the reconstructed position of the event. In other words, the longer the fluorescence decay time, the larger the uncertainties in the reconstructed position of the event. Re-emission times for the two satisfactory WLS compounds were measured with a laser system at Queen's University [6]. The measured re-emission times are shown in Figures 2.4 and 2.5. For Alexa Fluor, the re-emission time is $5.70 \pm 0.11$ ns, while for the Carbostyril the re-emission time was slightly higher at $6.17 \pm 0.09 \mathrm{~ns}$. 


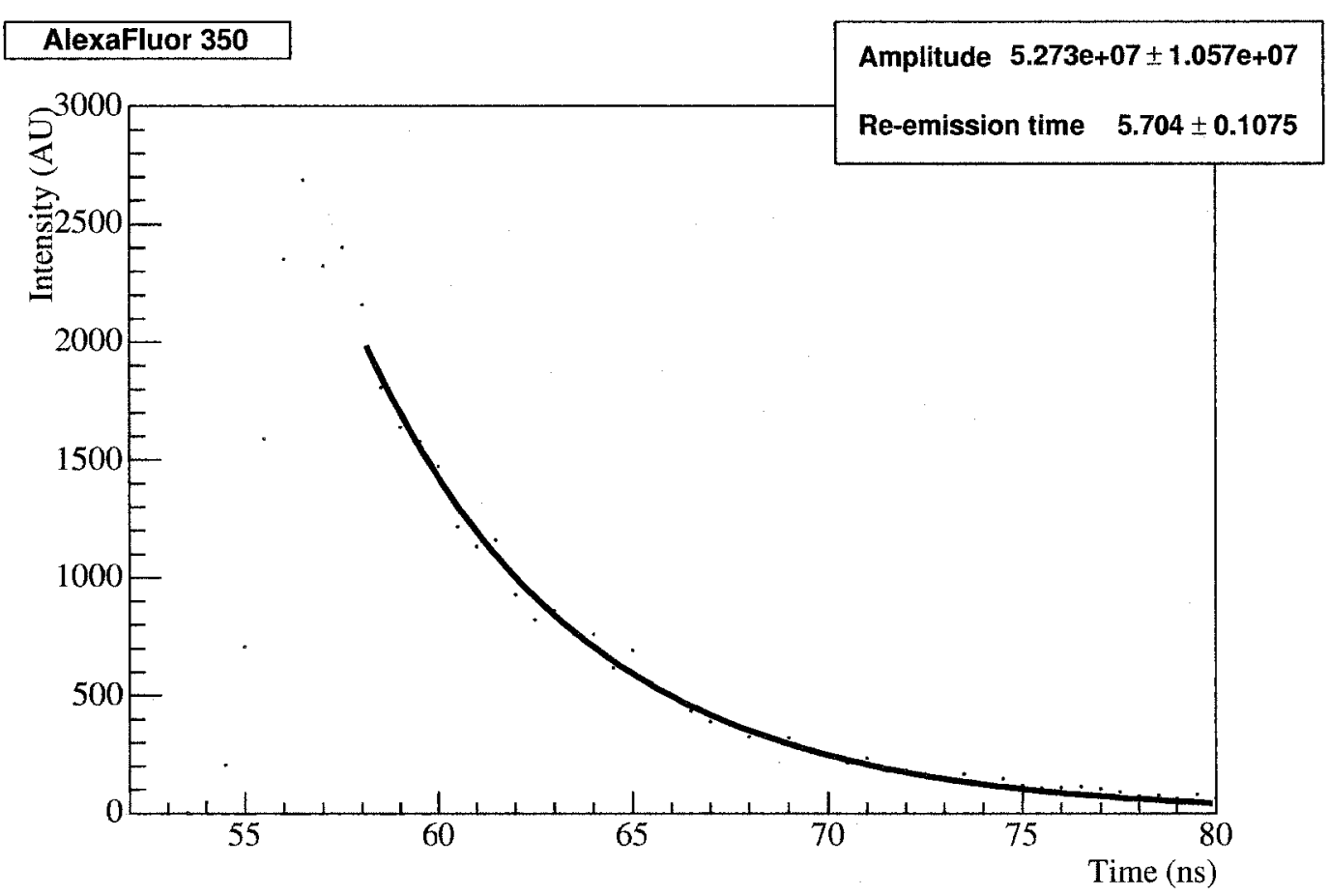

Figure 2.4: Intensity of the re-emission light as a function of time for the Alexa Fluor 350. The fitted line is an exponential decay function. 


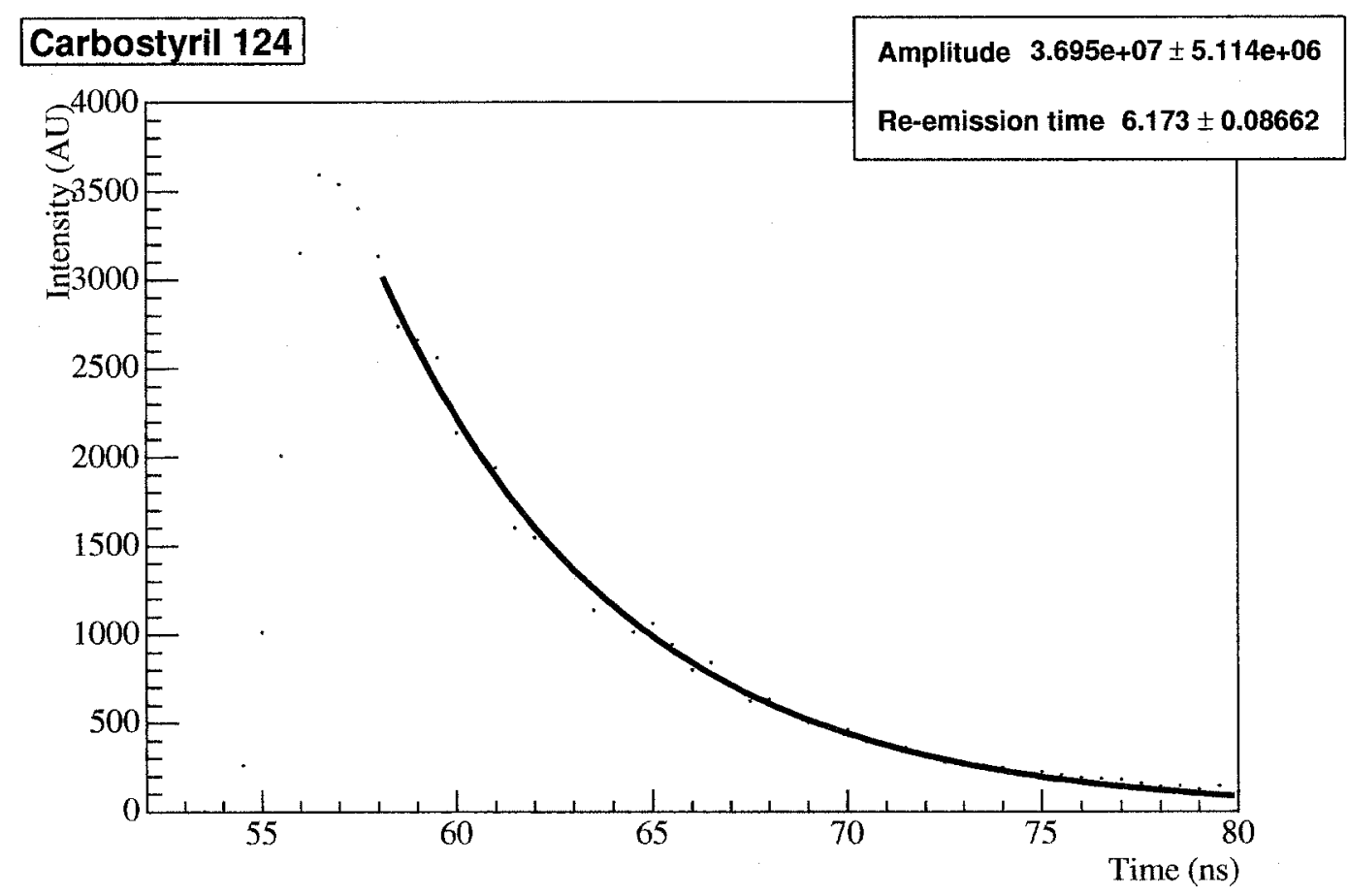

Figure 2.5: Intensity of the re-emission light as a function of time for the Carbostyril 124 . The fitted line is an exponential decay function. 


\begin{tabular}{|c||c|c|c|c|}
\hline $\begin{array}{c}\text { Candidate } \\
\text { name }\end{array}$ & $\begin{array}{c}\text { Quantum } \\
\text { Efficiency }\end{array}$ & $\begin{array}{c}\text { Re-emission peak } \\
\text { position }(\mathrm{nm})\end{array}$ & $\begin{array}{c}\text { Re-emission } \\
\text { time (ns) }\end{array}$ & $\begin{array}{c}\text { Price for } \\
500 \mathrm{mg}\end{array}$ \\
\hline \hline Alexa Fluor 350 & $92.8 \pm 0.5 \%$ & $444 \pm 1$ & $5.70 \pm 0.11$ & $\$ 300,000$ \\
Carbostyril 124 & $97.9 \pm 0.5 \%$ & $416 \pm 1$ & $6.17 \pm 0.09$ & $\$ 237$ \\
\hline
\end{tabular}

Table 2.1: Quantum efficiency, re-emission peak position, re-emission time and price for a small quantity of the Alexa Fluor and Carbostyril.

\subsection{Summary}

In conclusion, Alexa Fluor 350 and Carbostyril 124 are the only candidates that passed all viability tests. Carbostyril 124 seems to be the most appropriate WLS for several reasons: its absorption spectrum has no tail near $400 \mathrm{~nm}$ like the Alexa Fluor, its QE is slightly higher, and it is almost a thousand times cheaper. The properties of the Alexa Fluor and Carbostyril are summarized in Table 2.1. 


\section{Chapter 3}

\section{Monte Carlo Simulations for SNO}

\subsection{Parameters in SNOMAN}

Monte Carlo simulations were performed using the official SNO software, SNOMAN. The absorption spectrum, quantum efficiency, and wavelength of the re-emission peak were parametrized in order to recreate the interaction between photons and WLS. For each simulation, SNOMAN interpolated the absorption spectrum with a fifteen-point piecewise linear function. It also assumed a Gaussian distribution for the re-emission wavelength spectrum from an experimentally measured mean and width. Furthermore, SNOMAN used the input concentration to scale the absorption coefficient accordingly. 


\subsection{Determination of the WLS Concentration}

The first simulation was achieved in order to determine the right concentration of WLS to be used in SNO. The concentration values were chosen in the range from 0.01 to $5 \mathrm{ppm}$ based on the mean free path of ultraviolet photons in the detector. A high concentration maximizes the number of photons converted and reduces the uncertainty in the event position by shortening their mean free path. On the other hand, it is important to minimize the quantity of WLS for obvious financial reasons, and oversaturation of WLS could result in the possible absorption and re-emission cycles discussed in Section 2.2.3.

The simulation procedure was simple: 10,000 electrons with an energy of $10 \mathrm{MeV}$ were generated isotropically in the centre of the detector. The distribution of the number of hit PMTs (Nhits) is shown in Figure 3.1. The mean of the distributions is shown in Table 3.1.

At concentrations above $0.50 \mathrm{ppm}$, there was a saturation plateau in the number of detected photons. Therefore, there is no significant advantage in choosing a higher concentration in terms of Nhits. However, in order to keep the spatial resolution at a reasonable level and bring the mean free path of absorption below $10 \mathrm{~cm}$, the concentration should be higher or equal to $1 \mathrm{ppm}$. Simple calculations show that the delay of 6 ns caused by the fluorescence of the WLS can be interpreted as a length of one meter, and therefore dominates the mean free path position uncertainty at concentrations above $0.1 \mathrm{ppm}$. Therefore, the final choice of the concentration will depend on financial issues and on the improvements done on the event fitter at the time of the WLS phase of the experiment. The following discussion and analyses assumed a solution of $1 \mathrm{ppm}$ of Carbostyril 124. 


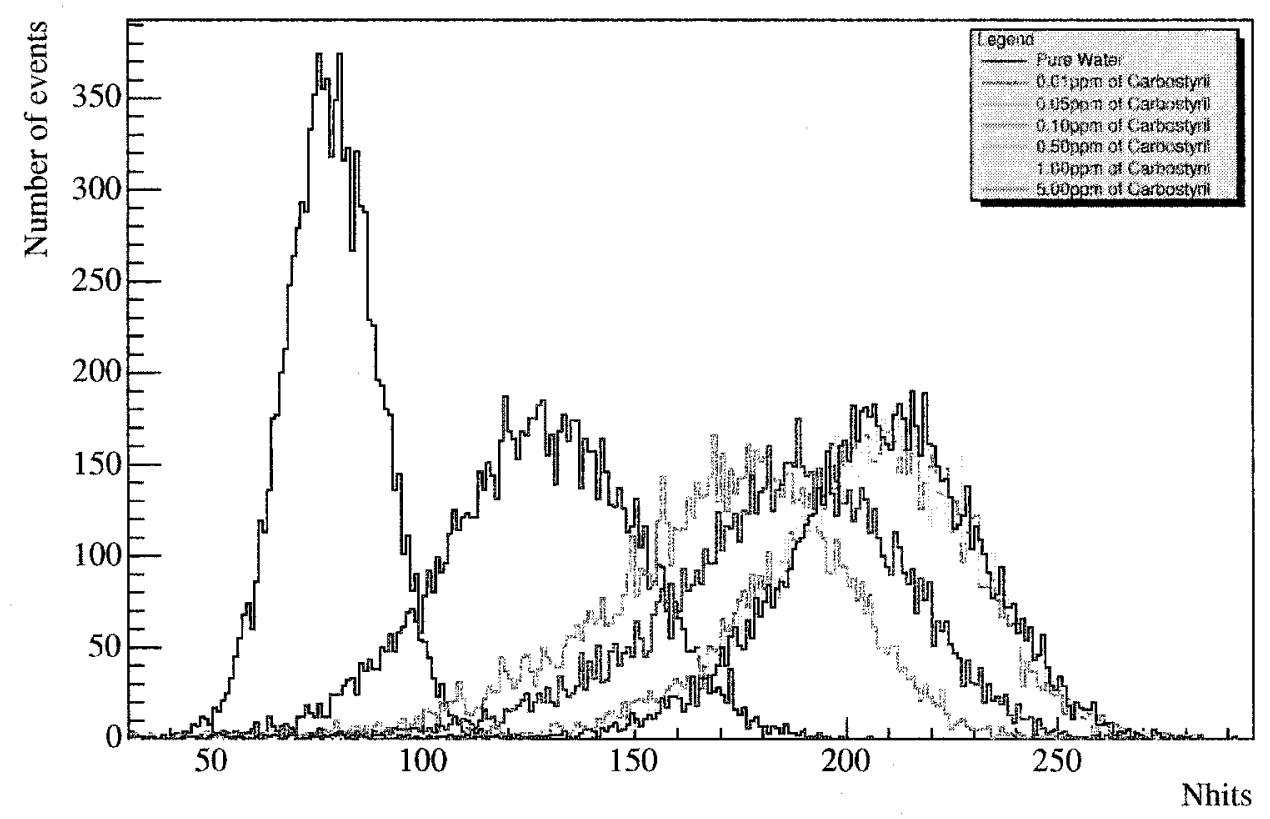

Figure 3.1: Nhits distribution as a function of the concentration of the WLS solution for $10 \mathrm{MeV}$ electrons. View this plot in color. 


\begin{tabular}{|c||c|c|c|}
\hline $\begin{array}{c}\text { WLS Concentration } \\
(\mathrm{ppm})\end{array}$ & $\begin{array}{c}\text { Mean Nhits } \\
\text { Alexa Fluor }\end{array}$ & $\begin{array}{c}\text { Mean Nhits } \\
\text { Carbostyril }\end{array}$ & $\begin{array}{c}\text { Mean Free Path } \\
\text { Approx. (cm) }\end{array}$ \\
\hline \hline 0.00 & $77.9 \pm 0.2$ & $77.9 \pm 0.2$ & $\infty$ \\
0.01 & $102.2 \pm 0.2$ & $126.4 \pm 0.2$ & 1000 \\
0.05 & $138.6 \pm 0.2$ & $167.8 \pm 0.2$ & 200 \\
0.10 & $156.7 \pm 0.2$ & $182.1 \pm 0.2$ & 100 \\
0.50 & $182.3 \pm 0.2$ & $201.9 \pm 0.2$ & 20 \\
1.00 & $187.7 \pm 0.2$ & $204.6 \pm 0.2$ & 10 \\
5.00 & $192.3 \pm 0.2$ & $205.9 \pm 0.2$ & 2 \\
\hline
\end{tabular}

Table 3.1: Mean Nhits as a function of the WLS concentration for both Alexa Fluor and Carbostyril. The measurements come from a Monte Carlo simulation that used 10,000 single $10 \mathrm{MeV}$ electrons created uniformly in the SNO detector and re-emitting photons isotropically. The last column shows an approximation of the mean free path for both candidates.

\subsection{Gain of Light}

\subsubsection{Analytical Approximation}

In order to compare the amount of light detected with and without WLS, an analytical approximation of the integral of the light below and above $300 \mathrm{~nm}$ has been done from Figure 2.1. The section below $300 \mathrm{~nm}$ represents the photons that would be absorbed by the acrylic, whereas the section above is associated with the original detected Čerenkov signal. This approximation gave a maximal theoretical light gain factor of 3.6, assuming a 100\% QE of the WLS and a $100 \%$ efficiency of the PMTs.

\subsubsection{Monte Carlo Dataset}

The gain from Monte Carlo was determined directly from the data in Table 3.1 that was used to determine the best concentration of WLS to be added 


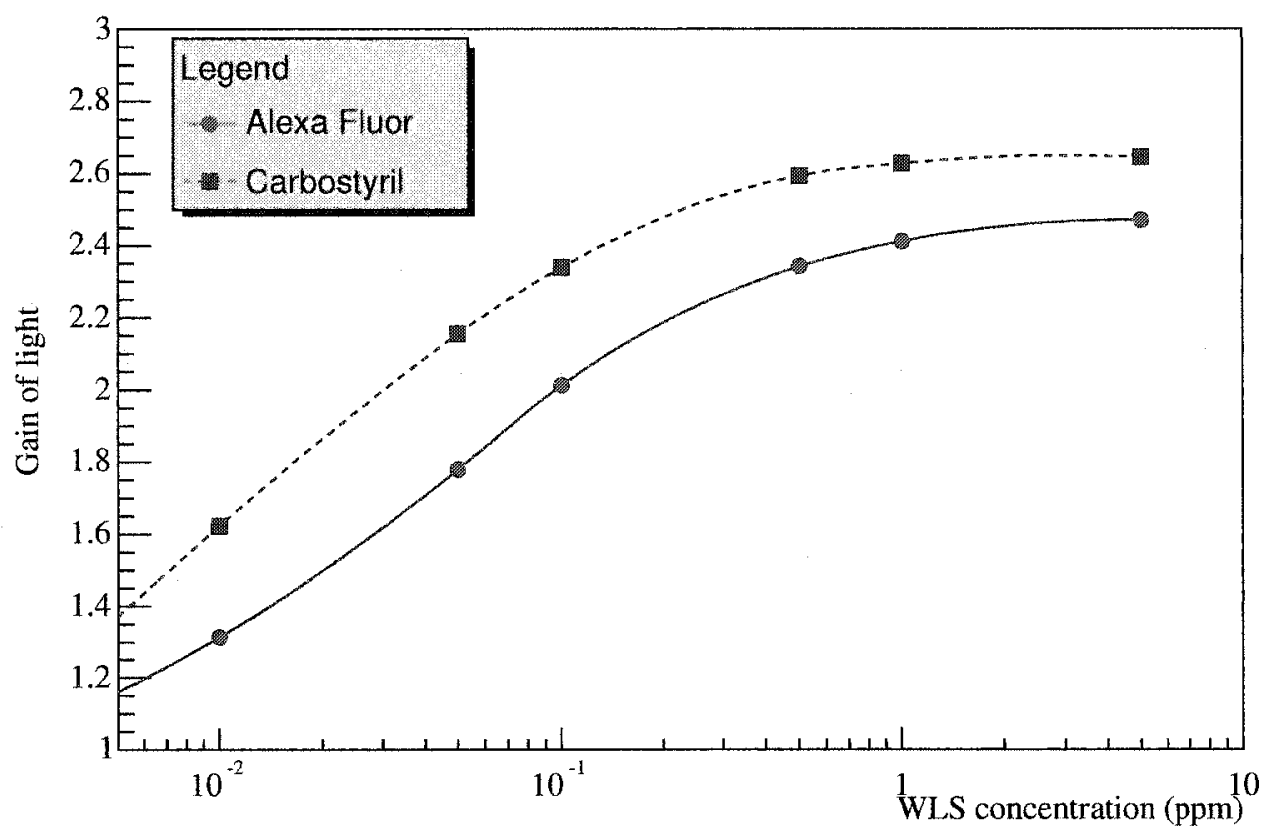

Figure 3.2: Gain of light as a function of the concentration of the WLS solution. The solid blue line represents the Alexa Fluor and the dotted red line corresponds to the Carbostyril.

to the SNO detector. There were 2.7 times more photons detected with a concentration of $1 \mathrm{ppm}$ of Carbostyril 124 than with pure $\mathrm{D}_{2} \mathrm{O}$. This is consistent with the analytical calculation mentioned in the previous section. The gains as a function of the concentration for both WLS candidates are plotted in Figure 3.2.

\subsubsection{Energy Resolution Uncertainty}

One of the dominant limitations in SNO's analysis is the energy resolution of the detector. One way to reduce this uncertainty would be to increase the number of Nhits per event, since the energy of an event is proportional to Nhits. 
Therefore, adding WLS to the SNO detector would improve the energy resolution.

\subsubsection{PMT Time distribution}

The PMT time distribution is used in SNO analyses to reconstruct the position and direction of an event. Since the fluorescence of WLS is approximately $6 \mathrm{~ns}$, a change in the time distribution of events is expected. Figure 3.3 shows this effect for different concentrations. Fortunatelly, the PMT hit time distribution seems to converge at concentrations greater than $0.05 \mathrm{ppm}$. To increase the precision of the event fitter used by SNO, the PMT time distributions should be updated with one of these distribution. This task is a complex one and is beyond the scope of this thesis.

\subsection{Significance of the Signal over the Back- ground}

Since the signal recorded by SNO is much more complex than the monoenergetic electrons considered in the last section, a better analysis would consist of comparing the simulated signals detected with and without WLS. One of the scientific quests of SNO is to investigate possible distortions of the $\nu_{e}$ energy spectrum caused by matter induced neutrino oscillations. Hence, the signal considered was the CC interaction with a low energy background wall. This background is caused by the natural ${ }^{208} \mathrm{Tl}$ to ${ }^{208} \mathrm{~Pb} \gamma$-transition at $2.615 \mathrm{MeV}$ and a $3.26 \mathrm{MeV} \beta$ from the decay of ${ }^{214} \mathrm{Bi}$ to ${ }^{214} \mathrm{Po}$. The number of $\mathrm{Tl}$ and $\mathrm{Bi}$ decays used for the simulation was taken from the $\mathrm{U}$ and $\mathrm{Th}$ concentration in 


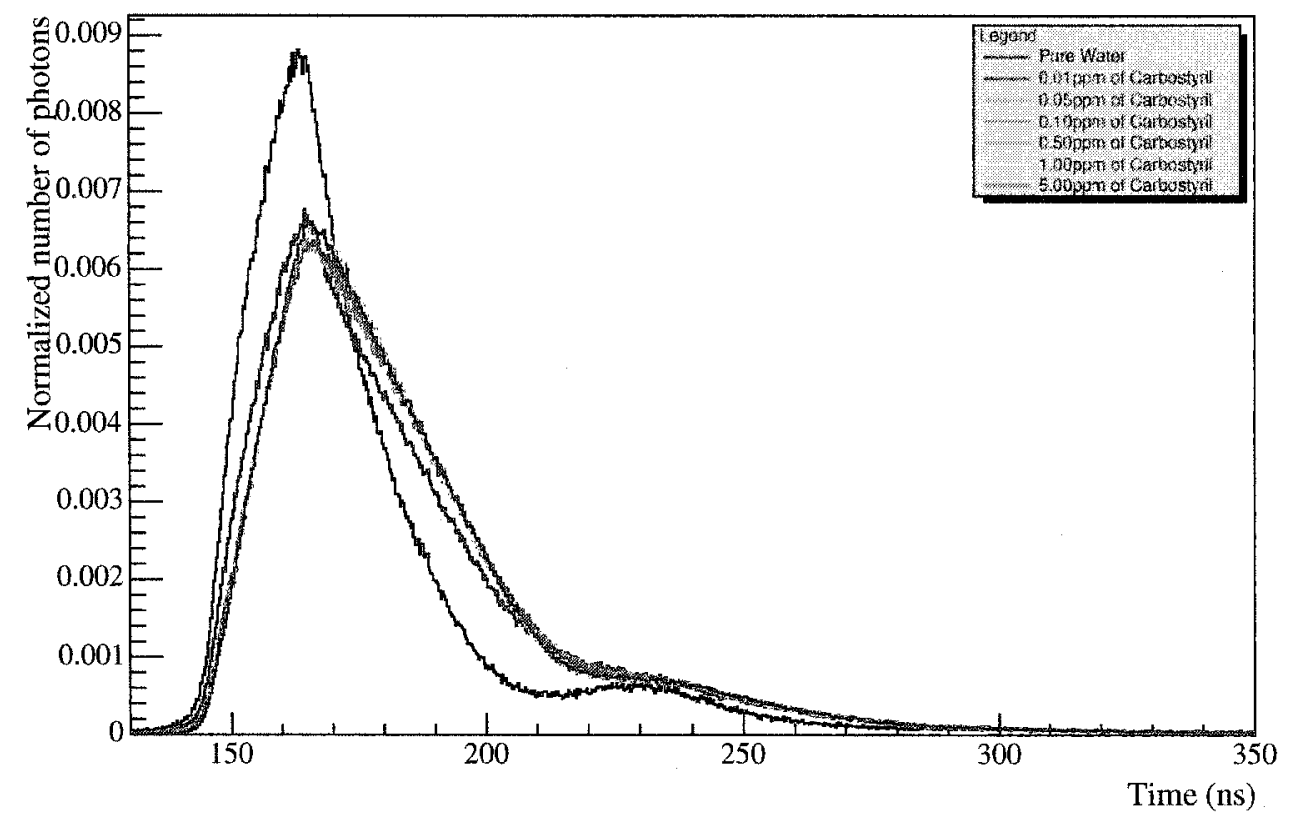

Figure 3.3: PMT time distribution as a function of the concentration of the WLS solution. View this plot in color. 
SNO, which are $6.0 \times 10^{-14} \mathrm{~g} / \mathrm{g}\left(\mathrm{D}_{2} \mathrm{O}\right)$ and $3.0 \times 10^{-15} \mathrm{~g} / \mathrm{g}\left(\mathrm{D}_{2} \mathrm{O}\right)$, respectively, in the $\mathrm{D}_{2} \mathrm{O}$. The low energy background distribution from outside the AV was parametrized from the distribution measured during the first phase of the experiment and corresponds to $1.9 \times 10^{-13} \mathrm{~g} / \mathrm{g}\left(\mathrm{H}_{2} \mathrm{O}\right)$ and $6.1 \times 10^{-14} \mathrm{~g} / \mathrm{g}\left(\mathrm{H}_{2} \mathrm{O}\right)$. Figures 3.4 and 3.5 show the Nhit spectra of both the signal and the background.

In the data analysis done by the SNO collaboration, an energy cut is usually applied to eliminate most of the background. A greater number of detected photons per event would allow an advantageous lower energy cut. First, many $\mathrm{CC}$ events would not be buried under the background wall. Approximatively $20 \%$ of the signal is between 3.7 and $5.5 \mathrm{MeV}$. A low energy cut would also decrease the statistical uncertainty on the measured flux. Second, it would provide access to a range of neutrino energies unanalyzed before by any solar neutrino experiment.

The energy cut was chosen to maximize the statistical significance of the signal (s) over the low energy background (b); both the efficiency $\left(\frac{s}{s_{\text {total }}}\right)$ and the purity $\left(\frac{s}{s+b}\right)$ have to be optimized. Consequently the figure of merit that is optimized to determine the energy threshold is the statistical significance of the signal over the background $\frac{s^{2}}{s+b}$. The best cut corresponds to an energy threshold of $5.1 \mathrm{MeV}$ for pure $\mathrm{D}_{2} \mathrm{O}$ and $3.7 \mathrm{MeV}$ for a $1 \mathrm{ppm}$ solution of Carbostyril as summarized in Table 3.2. Figures 3.4 and 3.5 show the position of the cut for each configuration discussed previously.

\subsection{Isotropy of the WLS Light}

Another property of WLS that was studied is the isotropy of the light it reemits. Since the directional information that is used to discriminate between the 

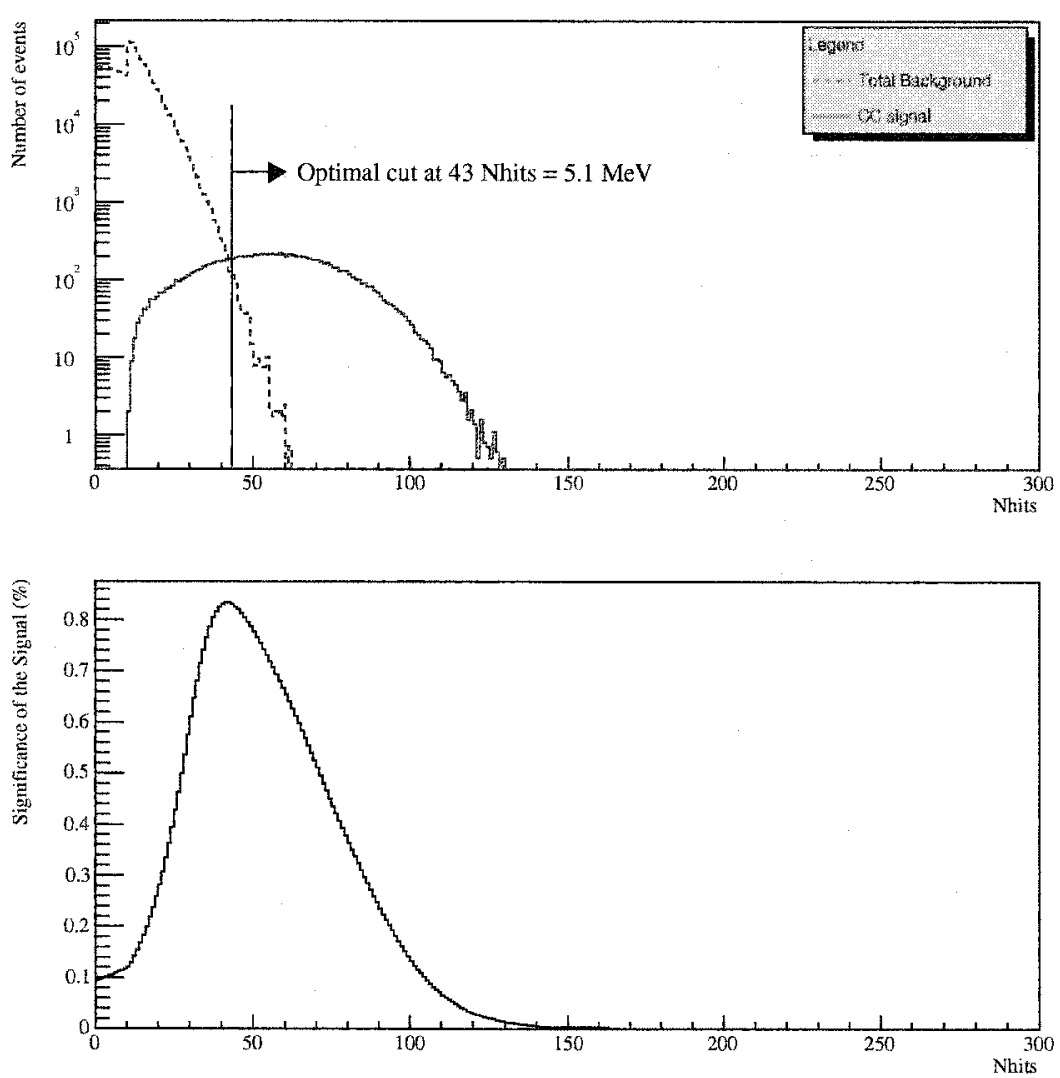

Figure 3.4: Energy spectra for the CC signal in dashed red and the background in solid blue without WLS for one SNO year with a $100 \%$ livetime. The optimal cut was found, as the most statistically significant signal over the low energy background, at $5.1 \mathrm{MeV}$. Lower: The figure of merit of the statistical significance of the signal defined as $\frac{s^{2}}{s+b}$. 

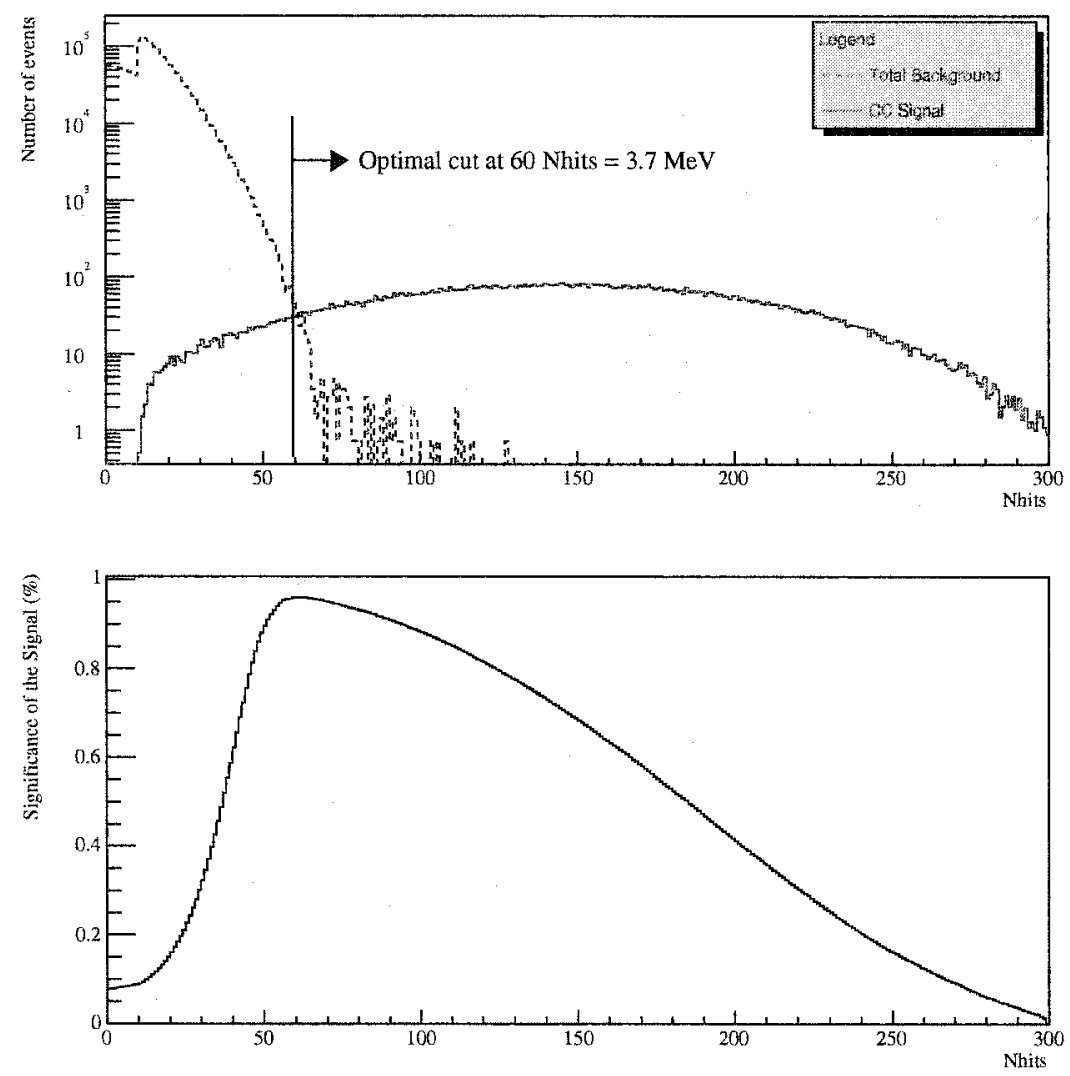

Figure 3.5: Upper: Energy spectra for the CC signal in dashed red and the background in solid blue with $1 \mathrm{ppm}$ of Carbostyril for one SNO year with a $100 \%$ livetime. The optimal cut was found, as the most statistically significant signal over the low energy background, at $3.7 \mathrm{MeV}$. Lower: The figure of merit of the statistical significance of the signal defined as $\frac{s^{2}}{s+b}$. 


\begin{tabular}{|c|c|}
\hline WLS name & $\begin{array}{c}\text { Energy Threshold } \\
(\mathrm{MeV})\end{array}$ \\
\hline \hline Pure $\mathrm{D}_{2} \mathrm{O}$ & 5.1 \\
Carbostyril at $1 \mathrm{ppm}$ & 3.7 \\
\hline
\end{tabular}

Table 3.2: Energy threshold that maximize the statistical significance of the signal for the pure $\mathrm{D}_{2} \mathrm{O}$ and the Carbostyril at $1 \mathrm{ppm}$.

three signals and the background is based on the cone pattern of the Cerenkov light, an increase in the uncertainties of the fit is expected as the concentration of WLS increases because the light is isotropic. To quantify this increase, a complex and much more detailed study would have to be done. For example, the current event fitter used by the SNO collaboration uses PMT time information to reconstruct the position of the event. Adding WLS would change this time distribution because of the late re-emitted WLS light and would increase the uncertainty on the fitted position of the event. The isotropic nature of the reemitted light could be used to triangulate the event position. The optimization of the event fitter is beyond the scope of this thesis and hence was not studied in detail. Fortunately, isotropy is not necessarily a disadvantage. It could increase the quality of the signal by making its spatial distribution more uniform and symmetrical.

\subsubsection{Uniformity}

Interactions that happen near the $\mathrm{AV}$ are difficult to reconstruct due to the refraction of photons by the acrylic at large angles. Figure 3.6 shows uniformly distributed $10 \mathrm{MeV}$ electrons inside the AV. The dotted red line is the reconstructed position in pure $\mathrm{D}_{2} \mathrm{O}$, while the solid blue line is that using 1 ppm of 
Carbostyril 124. There is a drop in the number of events reconstructed around $\left(R / R_{A V}\right)^{3}=0.6$, with the pure $\mathrm{D}_{2} \mathrm{O}$ configuration. Some of these events were reconstructed outside the $\mathrm{AV}$, showing the lack of information required for the fitter to work properly. When WLS were added, this problem was partially corrected; a radial cut on the volume performed to eliminate uncertainties on the signal could be relaxed. In addition to lowering the energy cut as discussed in Section 3.4, adding WLS would potentially allow an increase of the fiducial volume by facilitating the discrimination between signal and background near the $\mathrm{AV}$. It is instructive to note that the cut at 5.5 meters from the center of the $\mathrm{AV}$ eliminates $23 \%$ of all events, assuming a uniform distribution. Furthermore, pushing the fiducial cut to $\left(R / R_{A V}\right)^{3}=1$ allows one to remove the uncertainty on the position of the $\mathrm{AV}$, which is one of the dominant systematic uncertainties for the reconstruction of neutrino events.

\subsubsection{Symmetry}

\section{NCDs shadowing effect}

SNO's third phase consists of NCDs in the $\mathrm{D}_{2} \mathrm{O}$. They reduce the correlation between the measured $\mathrm{NC}$ and $\mathrm{CC}$ signals by identifying $\mathrm{NC}$ counts on an event-by-event basis, but they can also absorb or reflect Cerenkov light. This can decrease the number of detected photons. This effect is shown in Figure 3.7, where two distributions show a normalized number of Nhits as a function of the position for an electron beam propagating in the negative $\mathrm{z}$ direction. The normalization was done by scaling the distribution to have unity at $z=0$. Positive z positions mean that photons cross more NCD strings, thus reducing the Nhits. Conversely, the number of detected photons should be greater at negative 


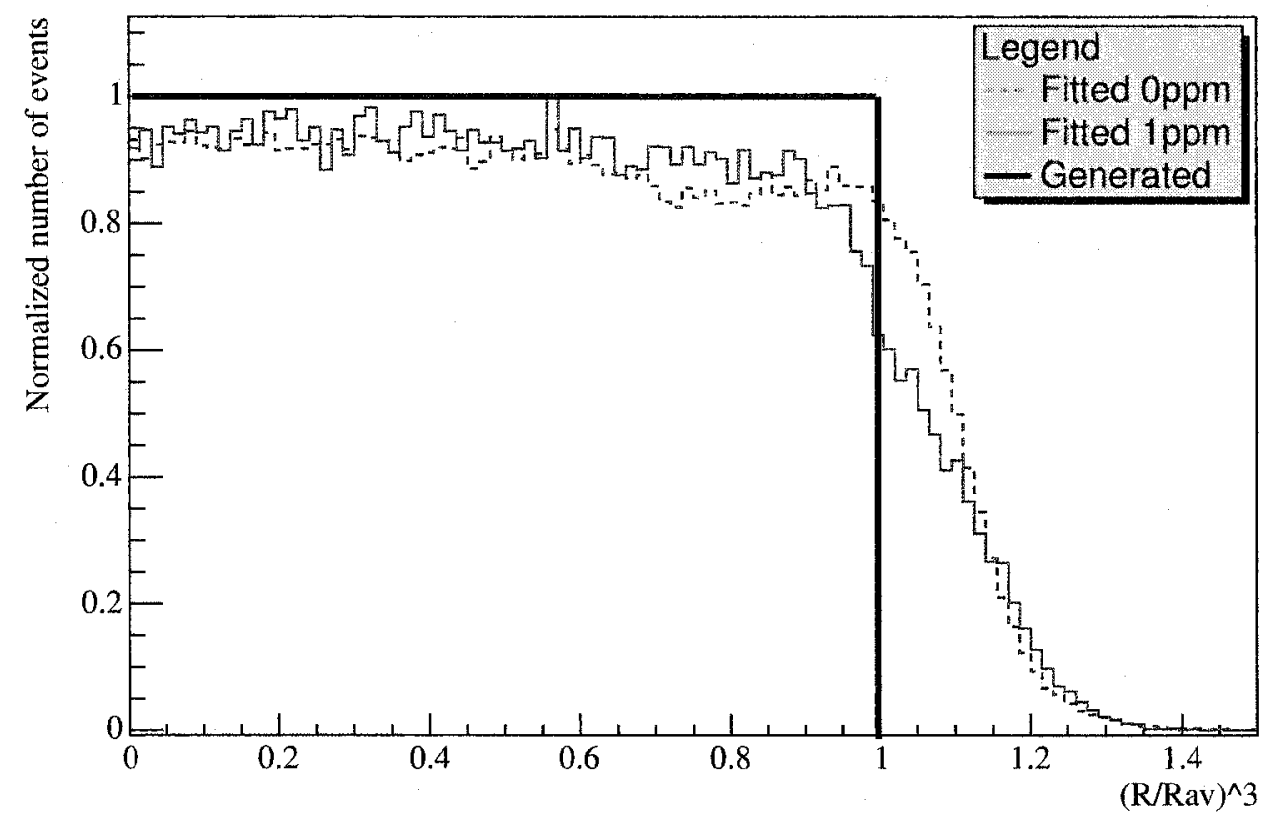

Figure 3.6: Uniformity of the signal after reconstruction. Electrons are generated uniformly inside the acrylic vessel represented by the black box. The dotted red line represents the normalized number of reconstructed event for each position for $\mathrm{D}_{2} \mathrm{O}$ configuration, while the solid blue line represents a concentration of 1 ppm of Carbostyril 124 . 
positions, close to the number of detected photons without NCD. The dotted red line in Figure 3.7 represents this effect in the absence of WLS. The $1 \mathrm{ppm}$ Carbostyril configuration, represented by the solid blue line, shows a more symmetrical distribution due to the isotropy of the re-emitted light. Adding WLS to the SNO detector would therefore decrease the shadowing effect of the NCDs.

\section{Energy scale uncertainty}

The energy scale of the SNO detector is obtained performing calibrations, therefore an uncertainty is associated to it. The energy scale uncertainty is a dominant one so reducing it would be an important achievement. As shown in the previous section, adding WLS to the $\mathrm{D}_{2} \mathrm{O}$ would make the SNO detector more symmetric and would make it easier to calibrate, thus decreasing the energy scale uncertainty.

\subsection{Overview}

According to Monte Carlo simulations, adding WLS to SNO's $\mathrm{D}_{2} \mathrm{O}$ would significantly improve the quality of the data by more than doubling the number of detected photons per event. This could reduce the energy threshold from 5.5 to $3.7 \mathrm{MeV}$ for a $\mathrm{CC}$ spectral analysis, make the fiducial volume larger and compensate for the shadowing effect and asymmetry of the NCDs. On the other hand, the Cerenkov radiation containing the directional information could be overwhelmed by the isotropic WLS light. Thus, modifying the SNO's current vertex fitter to take into account the fluorescence time of the WLS and the isotropic distribution of the light would be necessary for a WLS phase of SNO. 


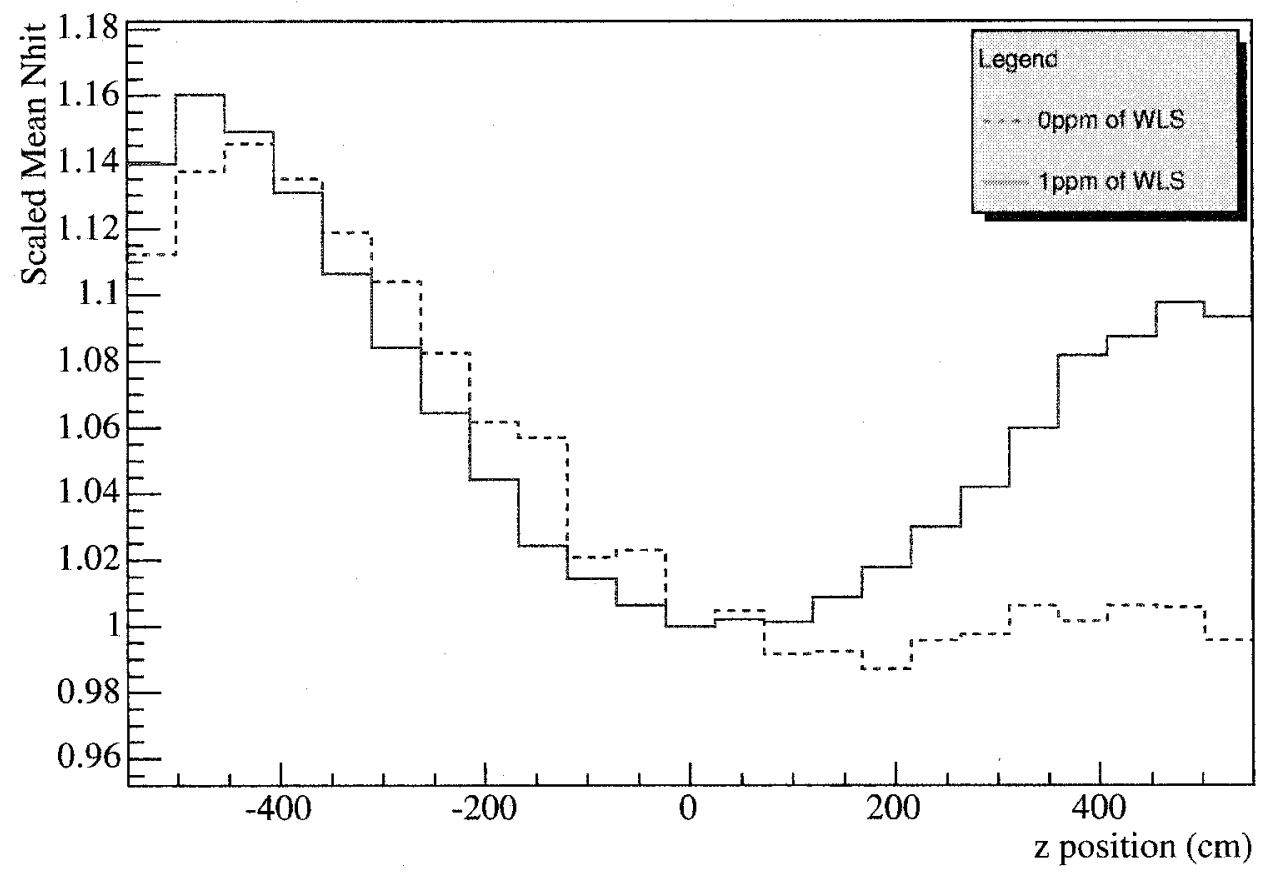

Figure 3.7: Symmetry of the signal with the NCD. Shown here is the number of detected photons as a function of the position of the generated electron. Electrons are propagated in the $-\mathrm{z}$ direction. The dotted red line represents the pure $\mathrm{D}_{2} \mathrm{O}$ configuration, while the solid blue line represents a configuration with a solution of $1 \mathrm{ppm}$ of Carbostyril. The Nhit value is scaled for each distribution to have unity at $\mathrm{z}=0 \mathrm{~cm}$. 


\section{Chapter 4}

\section{Neutrino Energy Spectrum}

This chapter describes SNO's sensitivity for detecting spectral distortions as a function of the two solar neutrino oscillation parameters $\delta m^{2}$ and $\tan ^{2} \theta$ for different detector configurations. As discussed in Chapter 3, a lower energy threshold of $3.7 \mathrm{MeV}$ is achievable if WLS is added to the $\mathrm{D}_{2} \mathrm{O}$. Such an improvement would give $\mathrm{SNO}$ a better sensitivity to neutrino energy spectrum distortions, which would give new information on matter induced oscillations. The current expectation is that there may be an excess of $\nu_{e}$ below $5.5 \mathrm{MeV}$ due to the lower probability of neutrino oscillation caused by matter as explained in references [7] and [8]. Measuring a distortion of the spectral shape would provide a strong evidence of this Mikheyev-Smirnov-Wolfenstein (MSW) effect responsible for neutrino flavour change. It is also expected to measure an asymmetry between the number of $\mathrm{CC}$ events detected during the day and night. Neutrinos detected during the night have to go through a larger amount of matter within the Earth, thereby possibly regenerating some $\nu_{e}$. 


\subsection{Neutrino Oscillation Formalism}

Neutrino oscillations depend on the electron density of the medium of propagation. Therefore, solar neutrinos oscillate differently in the Sun, in vacuum and in the Earth. To simplify the formalism, it is a good approximation to reduce the number of generations of lepton from three to two by merging the $\mu$ and $\tau$ flavours into one. Assuming an oscillation between weak eigenstates $\nu_{e}$ and $\nu_{\mu, \tau}$, the time evolution of a neutrino state is described by [10]:

$$
i \frac{d}{d t}\left(\begin{array}{c}
\left|\nu_{e}(t)\right\rangle \\
\left|\nu_{\mu, \tau}(t)\right\rangle
\end{array}\right)=M\left(\begin{array}{c}
\left|\nu_{e}(t)\right\rangle \\
\left|\nu_{\mu, \tau}(t)\right\rangle
\end{array}\right) .
$$

In vacuum,

$$
M=\frac{1}{4 p}\left(\begin{array}{cc}
-\delta m^{2} \cos 2 \theta & \delta m^{2} \sin 2 \theta \\
\delta m^{2} \sin 2 \theta & \delta m^{2} \cos 2 \theta
\end{array}\right),
$$

where $\delta m^{2}=m_{2}^{2}-m_{1}^{2}$ is the difference of mass squared between the two mass eigenstates $\nu_{1}$ and $\nu_{2}$ and $\theta$ the mixing angle. In matter, $\nu_{e}$ encounter a different potential than $\nu_{\mu, \tau}$, therefore

$$
M=\frac{1}{4 p}\left(\begin{array}{cc}
-\delta m^{2} \cos 2 \theta+2 p\left(V_{e}-V_{\mu, \tau}\right) & \delta m^{2} \sin 2 \theta \\
\delta m^{2} \sin 2 \theta & \delta m^{2} \cos 2 \theta
\end{array}\right),
$$

which can be rearranged into

$$
M=\frac{1}{4 p}\left(\begin{array}{cc}
-\delta m_{M}^{2} \cos 2 \theta_{M} & \delta m_{M}^{2} \sin 2 \theta_{M} \\
\delta m_{M}^{2} \sin 2 \theta_{M} & \delta m_{M}^{2} \cos 2 \theta_{M}
\end{array}\right)
$$

given

$$
\delta m_{M}^{2}=\sqrt{\left(\delta m^{2} \sin 2 \theta\right)^{2}+\left[\delta m^{2} \cos 2 \theta+2 p\left(V_{e}-V_{\mu, \tau}\right)\right]^{2}}
$$

and

$$
\theta_{M}=\frac{1}{2} \arctan \left(\frac{\tan 2 \theta}{1-\left[2 p\left(V_{e}-V_{\mu, \tau}\right) \delta m^{2}\right] \sec 2 \theta}\right)
$$


with $p$ being the magnitude of the momentum of the neutrino. $V_{e}$ and $V_{\mu, \tau}$ are the effective potentials that are given by

$$
\begin{gathered}
V_{e}=\sqrt{2} G_{F}\left(N_{e}-0.5 N_{n}\right), \\
V_{\mu, \tau}=\sqrt{2} G_{F}\left(-0.5 N_{n}\right),
\end{gathered}
$$

where $N_{e}$ and $N_{n}$ are the electron and neutron densities in matter and $G_{F}$ is the Fermi constant.

The mixing parameters $\delta m^{2}$ and $\theta$ are the physical inputs which determine the survival probability of the $\nu_{e}$. The probability that a $\nu_{e}$ stays in an electron state is

$$
P_{\nu_{e} \rightarrow \nu_{e}}=1-\frac{1}{2} \sin ^{2} 2 \theta\left(\text { or } \theta_{M}\right),
$$

for vacuum (or matter).

Equation 4.9 is modeled in the official SNO software, QPhysics, and is used to generate the survival probabilities with which both undistorted and distorted $\mathrm{CC}$ reconstructed energy spectra are calculated as a function of $\delta m^{2}$ and $\tan ^{2} \theta$. Figure 4.1 shows the CC spectra as simulated for 557.7 days of SNO data ${ }^{1}$ assuming $\delta m^{2}=7.1 \times 10^{-5} \mathrm{eV}^{2}$ and $\tan ^{2} \theta=0.39$, the preferred solution found in [9]. The red and the blue curves correspond to the day and the night distorted spectra, respectively. The black curve represents the undistorted spectra, scaled to the same number of events as the averaged day and night spectra. The thickness of the black curve represents the statistical uncertainty on the number of event per $1 \mathrm{MeV}$ bins. It is interesting to note that the three curves are very similar for $\delta m^{2}=7.1 \times 10^{-5} \mathrm{eV}^{2}$ and $\tan ^{2} \theta=0.39$.

\footnotetext{
${ }^{1}$ This corresponds to the number of days of data taking during the SNO $\mathrm{D}_{2} \mathrm{O}$ and Salt phases assuming a $55 \%$ livetime [9].
} 


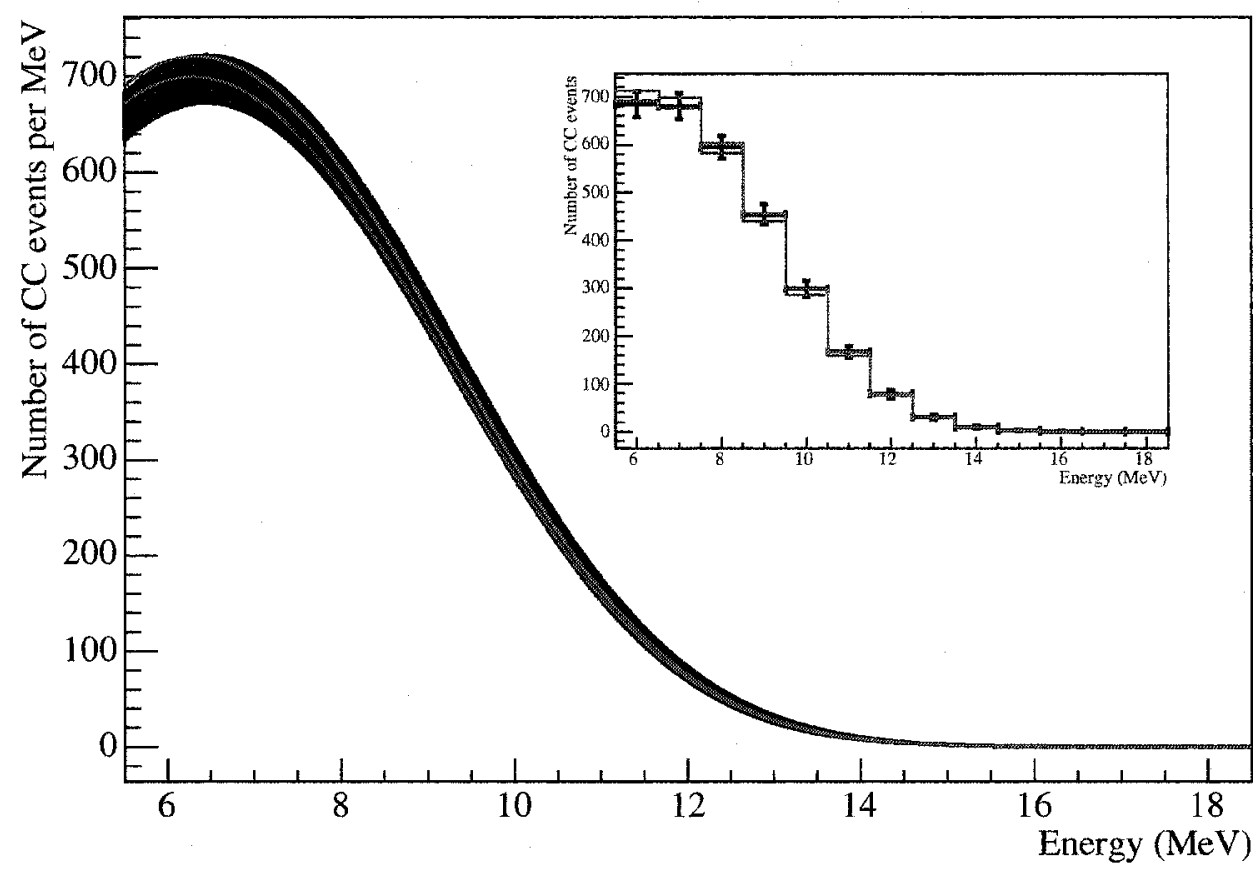

Figure 4.1: Simulated electron kinetic energy in CC events representing the duration of the $\mathrm{SNO} \mathrm{D}_{2} \mathrm{O}+$ Salt phases with 1014 days of data taking. The day (red) and night (blue) spectra were calculated with $\delta m^{2}=7.1 \times 10^{-5} \mathrm{eV}^{2}$ and $\tan ^{2} \theta=0.39$. This solution corresponds to the preferred solution from SNO's $\mathrm{D}_{2} \mathrm{O}+$ Salt data [9]. The thick black curve corresponds to undistorted ${ }^{8} \mathrm{~B} C \mathrm{CC}$ spectrum where the thickness of the band represents the statistical uncertainty. The plot on the upper right corner correspond to a close-up of the CC spectrum with $1 \mathrm{MeV}$ wide bins, similar to the one used in the $\chi^{2}$ test. The undistorted distributions were scaled to equal area with the rate predicted by the matter enhanced flavour changed. View this figure in color. 


\subsection{Test Statistic}

To quantify our ability to measure a spectral distortion, a test statistic was constructed. The test statistic is defined as:

$$
\chi^{2}=\sum_{i=1}^{N} \frac{\left(a_{i}-\nu_{i}\right)^{2}}{\nu_{i}}
$$

where $N$ is the total number of energy bins, $a_{i}$ is the total number of CC events reconstructed in bin $i$ for a given $\delta m^{2}$ and $\tan ^{2} \theta$, and $\nu_{i}$ is the expected number of CC events in $i$ under the hypothesis of an undistorted energy spectrum. Here the null hypothesis $H_{0}$ is an undistorted spectrum. The test statistic follows a $\chi^{2}$ distribution with $N$ degrees of freedom under the null hypothesis. Consequently, the P-value of the test statistic is expected to follow a uniform distribution if the null hypothesis is correct.

A similar test can be performed exploiting the asymmetry between the day and night spectra. In this case the test statistic becomes:

$$
\chi^{2}=\sum_{i=1}^{N} \frac{\left(d_{i}-\nu_{i}\right)^{2}}{\nu_{i}}+\sum_{i=1}^{N} \frac{\left(n_{i}-\nu_{i}\right)^{2}}{\nu_{i}}
$$

where $a_{i}$ is replace by $d_{i}$ or $n_{i}$ for the number of CC events in the day bin $i$ or the night bin $i$. Note that Equations 4.10 and 4.11 only test the shape of the spectra and not the rate of neutrino production.

\subsection{Spectral Distortion Analysis}

The goal of this study is to find if spectral distortions can be detected for different configurations of the SNO detector. The oscillation parameter space was divided into a grid of two hundred by two hundred points and, using the 
test statistic defined in Section 4.2, each point was tested for SNO's sensitivity to spectrum distortions. Unfortunately, this technique had limitations. It was assumed that all three signals and the background would be disentangled with a $100 \%$ efficiency. Since different types of events cannot be differentiated on an event-by-event basis, a fit must be done and the related uncertainty is difficult to predict and was not accounted for in this analysis. Therefore, the results quoted in the next sections assume a perfect separation of $\mathrm{CC}$ events from other and should be quoted as an optimistic view of the capability to detect spectral distortions.

\subsection{1 $\quad$ 5.5 MeV Energy Threshold}

\section{Procedures}

The first case considered has an energy threshold of $5.5 \mathrm{MeV}$, like the one used in the $\mathrm{D}_{2} \mathrm{O}$ and Salt SNO analyses. Bins are arbitrary determined to be $1 \mathrm{MeV}$ wide, from the threshold to $15.5 \mathrm{MeV}$, where the number of event becomes negligible. The simulated spectrum is composed of 1014 days of data taking with a $55 \%$ livetime and a fiducial volume cut at 5.5 meters, meaning that only $77 \%$ of the $\mathrm{D}_{2} \mathrm{O}$ volume was analyzed. The two largest uncertainties of the detector were considered in the statistical test.

The first uncertainty was related to the energy scale. It was simulated by shifting $2 \%$ of the content of each bin to the following one and re-calculating the $\chi^{2}$ for this scenario. Then, the same procedure was done but events were shifted to the previous bin. Finally, the $\chi^{2}$ was also calculated without any shift. For each of the MSW grid defined previously, three different $\chi^{2}$ values were available for the analysis and to keep a conservative approach, the lower value of the $\chi^{2}$ 
was chosen to account for the intrinsic energy uncertainty of SNO.

The second uncertainty included in the analysis is a $20 \%$ uncertainty in the number of background events. The majority of the background events happens near the energy threshold as depicted in Figures 3.4 and 3.5. A quick study showed that in the first $1 \mathrm{MeV}$ bin, the uncertainty on the background would always give a $100 \%$ consistency between the distorted and undistorted CC spectra. However, the following bins contained a negligible background signal. For simplicity, the first bin was not taken into account in the sum of the $\chi^{2}$, but was considered as a degree of freedom.

\section{Results}

Figure 4.2 shows an oscillation parameter plot with regions where the undistorted spectrum hypothesis is excluded at a $99.73 \%$ confidence level (C.L.). Two colored regions are shown on this figure: one is filled with a dark red color using the general test statistic described in Equation 4.10, the other one is filled with a light green color, using the day-night asymmetry in Equation 4.11. Superimposed on those regions are the confidence level contours of the $\mathrm{SNO} \mathrm{D}_{2} \mathrm{O}+\mathrm{Salt}$ phases at 90, 95, 99 and $99.73 \%$ C.L. published in [9]. As expected, the best fit point (star marker) is in the middle of a white region, so the simulated dataset did not have enough events to see spectral distortions in the CC signal. It is worthwhile to note that the level of distortion is different during the day and during the night for some regions of $\delta m^{2}$ and $\tan ^{2} \theta$. A large experimentally accessible region appears around $\log _{10}\left(\delta m^{2}\right)=-5.5$ and $\log _{10}\left(\tan ^{2} \theta\right)=-0.5$ when day and night spectra are taken separately. The separation of both spectra reduces the statistics in each spectrum by a factor of two, but increases the sensitivity to distortions near the bottom of the preferred region. Note that only 
the spectral shape is tested for, not the absolute rate.

According to the MSW theory, day-night asymmetry is larger at high energy. Unfortunately, as seen in Figure 4.1, the small number of events makes this effect difficult to observe. Nevertheless, spectra with the best fit oscillation parameters do show an asymmetry; this justifies a lower energy threshold. According to this analysis, the day-night asymmetry needs to be studied in order to constrain a larger region of the MSW plane.

\subsection{2 $\quad$ 3.7 MeV Energy Threshold}

\section{Procedures}

A lower energy threshold would increase the sensitivity to spectral distortion since close to the best fit oscillation parameters, there are larger distortions at low energies (c.f. Figure 4.1). Equivalently, reducing the energy threshold reduces the numbers of years of data taking needed to claim the discovery of spectral distortion caused by matter enhanced neutrino flavour change.

As seen in Chapter 3, an energy cut at $3.7 \mathrm{MeV}$ would be achievable using a Carbostyril solution of $1 \mathrm{ppm}$. It has also been demonstrated that the cut on the fiducial volume could be relaxed, but to keep a conservative approach the same cut at 5.5 meters from the center of the detector was assumed. Dead time due to calibrations, maintenance or other unfortunate activities was unknown, so the livetime was assumed to be $100 \%$. As a result, a year is 365.25 complete days of data. As before, the two most important systematic uncertainties were included in the analysis to make it more realistic.

As the number of years of data taking is unknown, it was kept variable. Hence, the format chosen to show the sensitivity to spectrum distortion of a WLS 


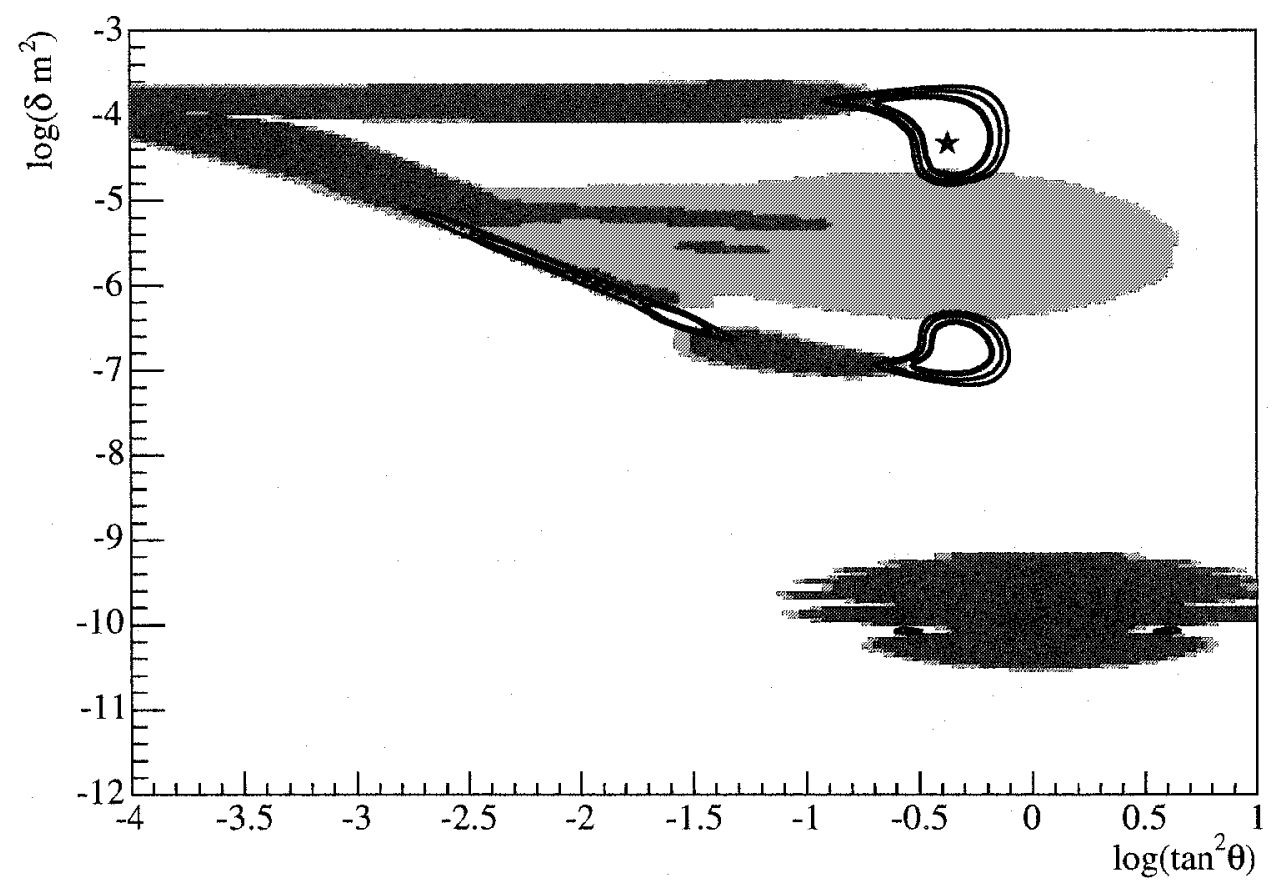

Figure 4.2: Region of the neutrino oscillation plane which excludes the undistorted spectrum hypothesis at a $99.73 \%$ C.L. $(3 \sigma)$. The simulated signal correspond to a duration equivalent to the $\mathrm{SNO} \mathrm{D}_{2} \mathrm{O}+$ Salt dataset. The dark red region excludes the undistorted hypothesis with the averaged day-night spectrum, while the light green region uses the day-night asymmetry. Superimposed are the $90,95,99$ and $99.73 \%$ C.L. contours using SNO published $\mathrm{D}_{2} \mathrm{O}+$ Salt data only [9]. View this plot in color. 
phase is defined as follows: for each set of $\delta m^{2}$ and $\tan ^{2} \theta$ values, the number of years of data taking required to claim the rejection of the no distortion hypothesis at $99.73 \%$ C.L. difference between distorted and undistorted distributions is calculated. The number of years is shown with a color code from purple ( $<1$ year) to red ( $>10$ years)

\section{Results}

Figure 4.3 shows how well the implication of a SNO WLS phase can measure spectral distortions with the systematic uncertainties discussed. The striking feature is that the number of years for rejecting $H_{0}$ increases dramatically near the boundary allowed by the $\mathrm{D}_{2} \mathrm{O}+$ Salt global fit. Hence, it would be hard to reject the undistorted spectrum hypothesis within the preferred region. If one assumes that SNO's best fit $\left(\delta m^{2}, \tan ^{2} \theta\right)$ are the true values, it would take 7.1 years with $100 \%$ livetime and $3.7 \mathrm{MeV}$ energy threshold to reject the null hypothesis at $99.73 \%$ C.L.

\subsection{MeV Energy Threshold}

\section{Procedures}

The addition of Carbostyril 124 molecules in $\mathrm{D}_{2} \mathrm{O}$ allows the reduction of the energy threshold to $3.7 \mathrm{MeV}$, but some other hypothetical configurations could lower this threshold further. So, to have a perfect experiment and an ideal detector, the systematic uncertainties were removed, the radial cut was lifted, and a $100 \%$ lifetime was assumed. In addition, the statistical test was modified to use only two bins, one from 3.25 to $7.00 \mathrm{MeV}$ and another from 7.00 to $10.75 \mathrm{MeV}$. It was found to be one of the most sensitive test statistic 


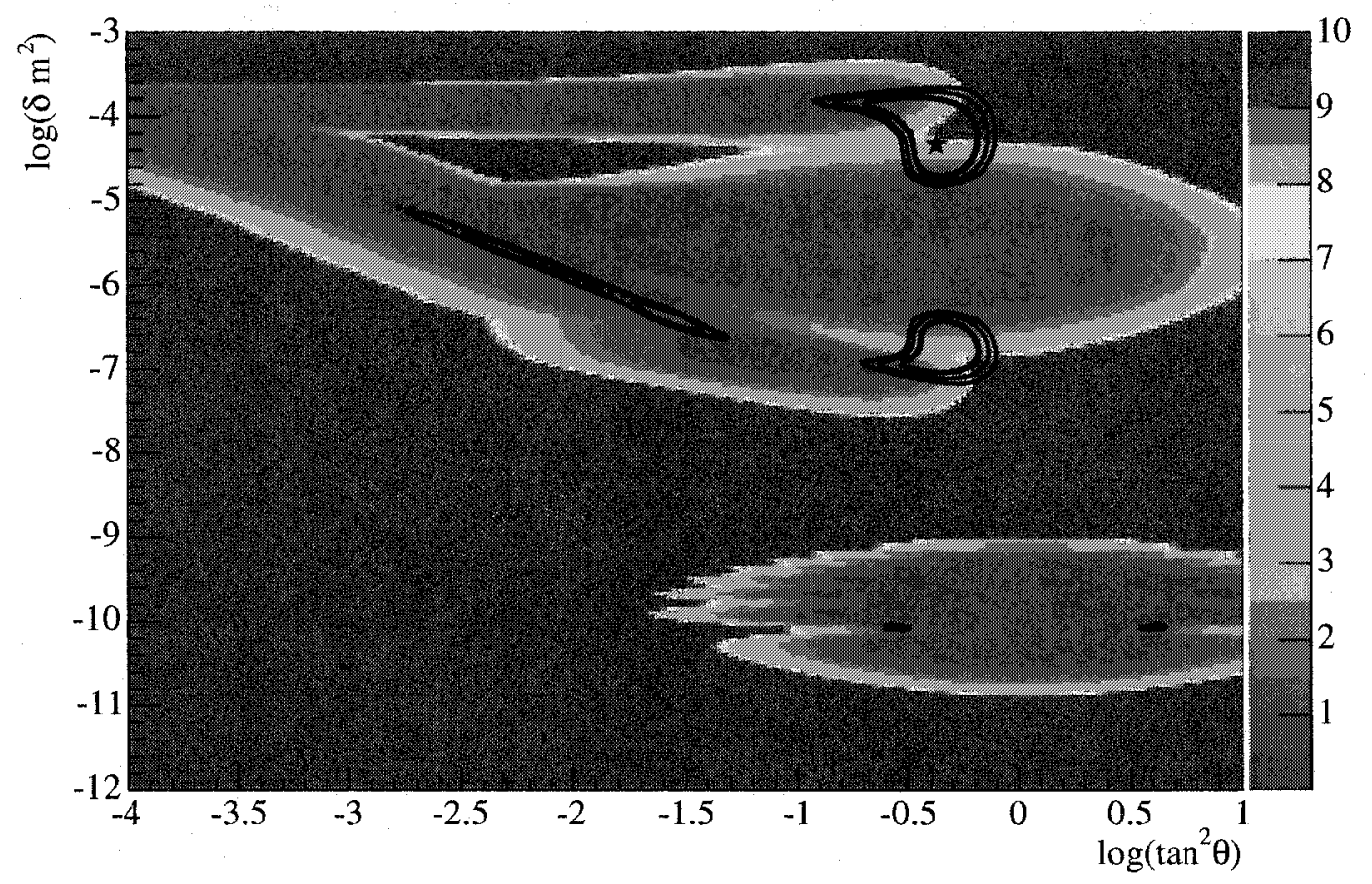

Figure 4.3: Number of years before excluding the undistorted spectrum hypothesis at $99.73 \%$ C.L. $(3 \sigma)$ for an energy threshold of $3.7 \mathrm{MeV}$. The current constrained regions using SNO data only with the 90, 95, 99 and $99.73 \%$ C.L. contours are superimposed on the MSW plane. View this plot in color. 
assuming SNO's best fit values for $\delta m^{2}$ and $\tan ^{2} \theta$. Therefore, it is the ultimate best case scenario for SNO.

\section{Results}

The global picture seems to change significantly as seen on Figure 4.4. The difference is large when one is looking at the region near SNO's preferred solution. The amount of data required to reject the null hypothesis at $99.73 \%$ C.L. changes rapidly as a function of $\left(\delta m^{2}, \tan ^{2} \theta\right)$ near the best fit point. Since the value of the low energy threshold affects the location of rapid change in sensitivity, lowering the energy threshold may yield the discovery of spectral distortions caused by matter effects.

In conclusion, the investigation of spectral distortions in the preferred region requires a low energy threshold, an excellent energy resolution, a very good understanding of the low energy backgrounds and the elimination of systematic uncertainties. For any configuration, it would be hard to analyze data below $3.25 \mathrm{MeV}$ because of the $3.24 \mathrm{MeV} \gamma$ from the Uranium decay chain.

\subsection{Summary}

This study shows that investigation of the day and night shapes of the CC energy spectrum provides constraints on the neutrino oscillation parameters. These constraints are complementary to the constraints obtained from measuring the neutrino fluxes. Near the preferred solution, the rejection of the null hypothesis is a challenge. Precise measurements of the day-night asymmetry are essential to constrain the low $\delta m^{2}$ part of the preferred region, while the low energy CC spectrum constrains the large $\delta m^{2}$, small $\tan ^{2} \theta$ part of the preferred 

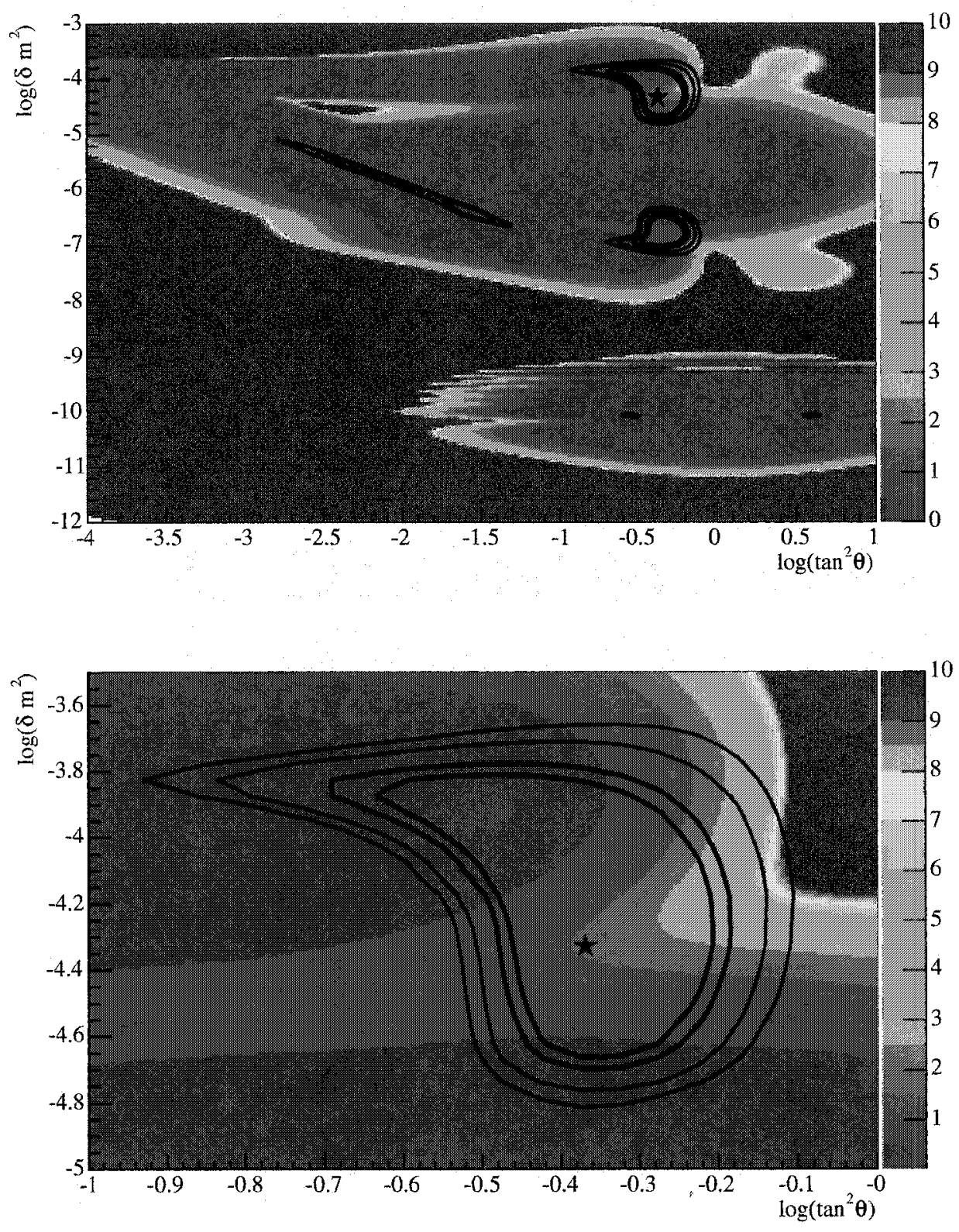

Figure 4.4: Number of years before excluding the undistorted spectrum hypothesis at $99.73 \%$ C.L. $(3 \sigma)$ for an energy threshold of $3.25 \mathrm{MeV}$ in the absence of systematic uncertainties. The current constrained regions using SNO data only with the 90, 95, 99 and $99.73 \%$ C.L. contours are superimposed on the MSW plots. A zoom on the preferred region is depicted in the lower plot. View these plots in color. 
region. Both measurements are needed to obtain the best constraints on the allowed region of the oscillation parameter space. 


\section{Chapter 5}

\section{Wavelength Shifter Cosmic Ray}

\section{Telescope}

Following the Monte Carlo studies, more measurements were needed to completely understand the advantages of adding WLS to Čerenkov detectors and to validate the results concerning the properties of WLS. A WLS cosmic ray telescope was identified as the necessary experimental apparatus to conduct optical and temporal measurements on WLS molecules. The general idea was to use optical media similar to those encountered by photons in the SNO experiment and hence reproduce the detector response to Cerenkov light. The WLS cosmic ray telescope was designed and constructed in the summer of 2003 and fully commissioned in 2004.

The challenging experimental unknown is the investigation of the possible absorption of very short wavelength photons by WLS. The absorption spectrum of WLS below $200 \mathrm{~nm}$ is unknown due to the limitations of commonly used commercial spectrometers. There is a large amount of Čerenkov light emitted below that range and since the Monte Carlo simulation discussed in Chapter 3 did 
not consider it, a measurement of the quantity of light re-emitted by WLS and its timing distribution were required. Studying these high energy photons is viewed as an interesting and novel development for water based neutrino detectors.

\subsection{Description of the Apparatus}

\subsection{1 Čerenkov Cells}

The design of the WLS telescope (see Figure 5.1) allows the direct comparison of WLS and Čerenkov photons produced by atmospheric muons. The main components of the telescope are two cylindrical Cerenkov polyvinyl chloride $(\mathrm{PVC})$ cells, with a height of $15 \mathrm{~cm}$ and a radius of $10 \mathrm{~cm}$. They are filled with a dielectric medium - water, WLS solution or liquid scintillator-, and are separated from an adjacent Hamamatsu R1408 SNO's PMT by an acrylic window. The thickness of the windows can be varied to understand the effect of acrylic on ultraviolet photons. Available thicknesses are $1.27,2.54$ and $5.08 \mathrm{~cm}$. Depending on the orientation of the cells, the PMTs could detect either direct Črenkov light, re-emitted WLS light or both, as shown in Figure 5.2. Two rubber hoses attached to a peristaltic pump were used to fill and empty the cells on a time scale of an hour. For stability, both cells are mounted on a metal frame and they can be easily flipped to change the configuration. A layer of mu-metal covers the side of each cell to shield the PMTs from magnetic fields. The PMT gain is affected by the Earth's magnetic field. Since the metallic frame is mobile, proper shielding reduces the variation of the gain due to this effect. 


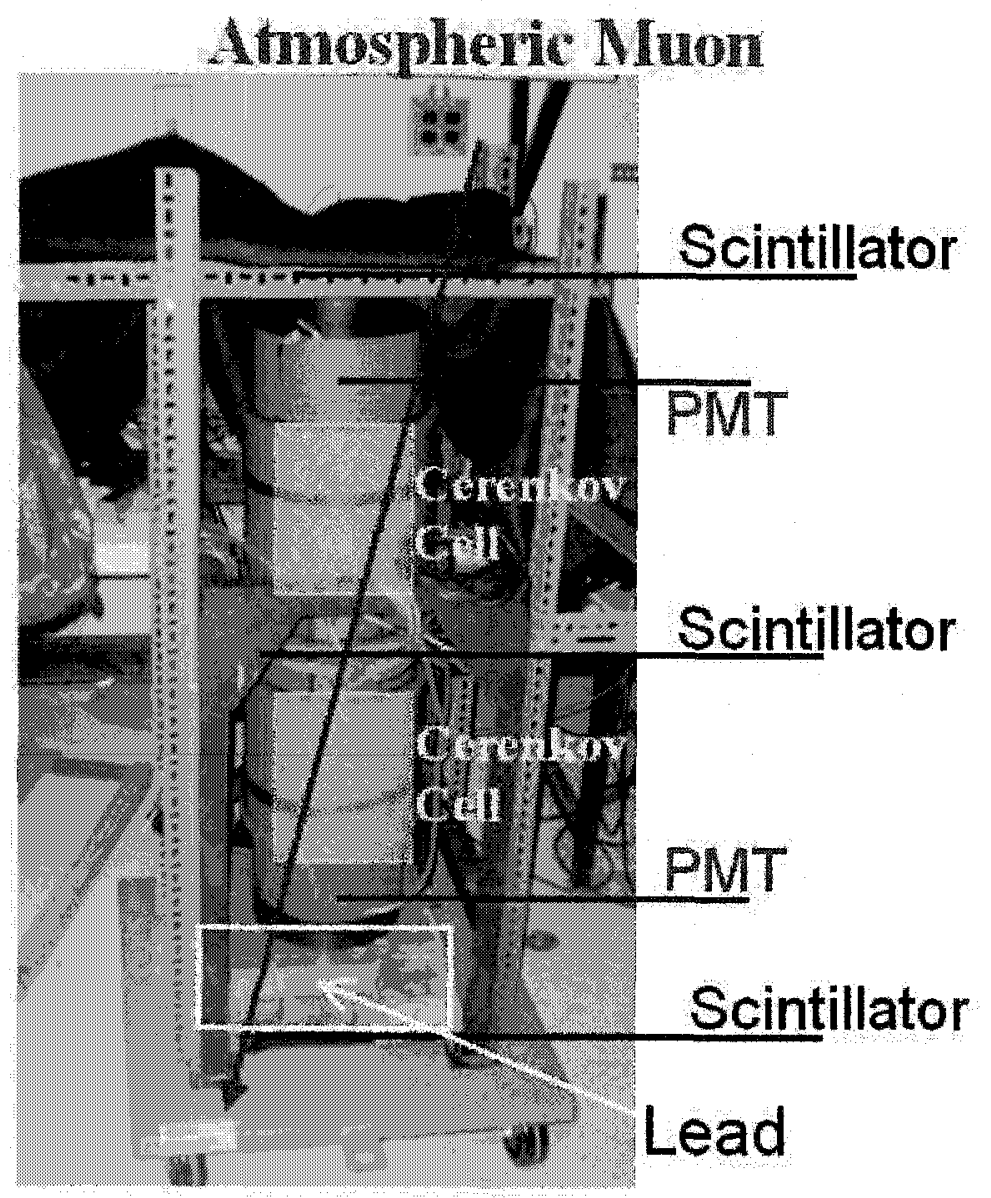

Figure 5.1: View of the WLS telescope. The total height of the telescope is about $1.5 \mathrm{~m}$. 

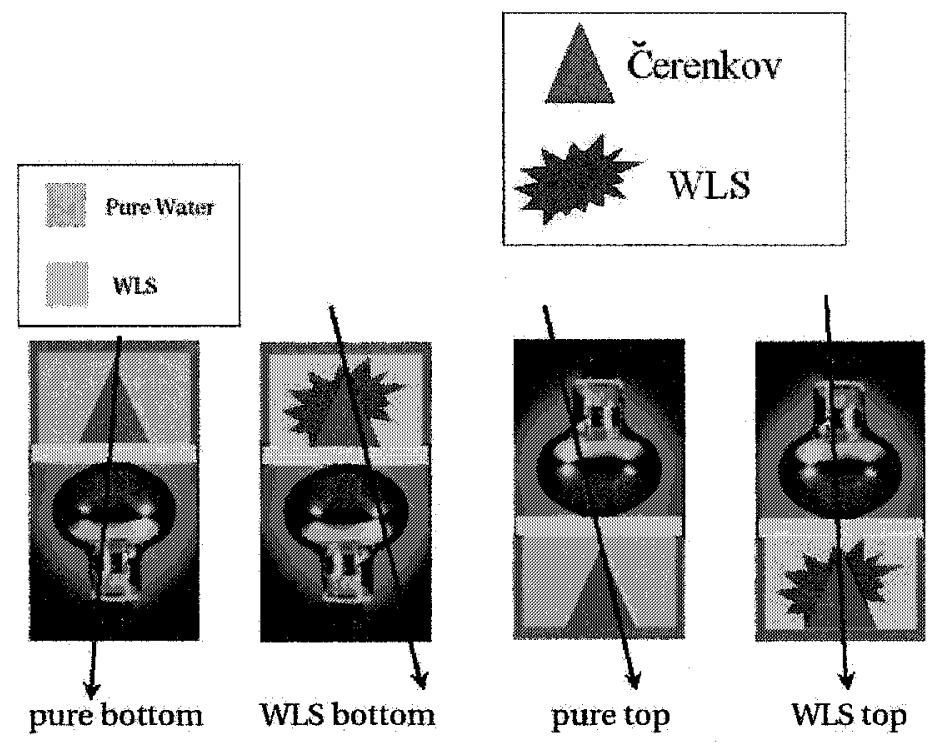

Figure 5.2: Different configurations for data taking of the WLS telescope and the associated detector response to Cerenkov and WLS radiations. Each cell are cylindrical with a $15 \mathrm{~cm}$ height and a $10 \mathrm{~cm}$ radius. View this figure in color. 


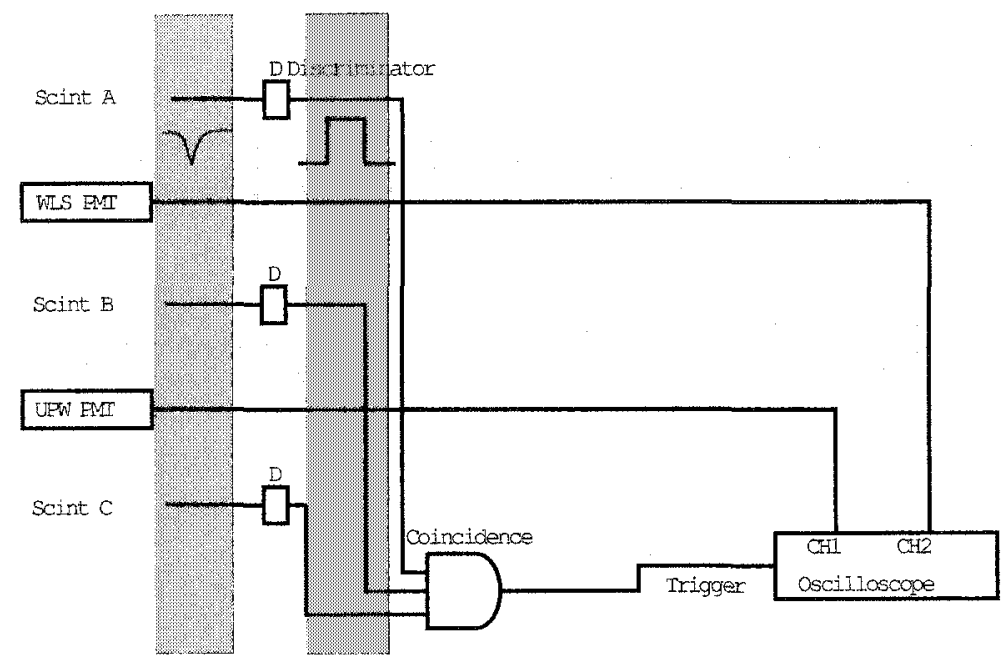

Figure 5.3: Logic circuitry of the WLS telescope.

\subsubsection{Scintillators}

A system of three scintillators, placed at the top, centre and bottom of the detector, triggered when a relativistic charged particle goes through all three scintillators. Relativistic charged particles passing through the scintillator are detected by scintilation light. If the electrical pulse from the scintillator is above the threshold of a discriminator that is attached to it, a second pulse is sent to a coincidence box. If all three scintillators generate a pulse within $20 \mathrm{~ns}$ of each other, a trigger signal is transmitted to a digital oscilloscope that records the waveforms of the two PMTs. The logic circuitry is depicted in Figure 5.3.

There are two distinctive features related to scintillators. The middle scintillator is smaller than the others in order to fit the diameter of the Cerenkov cells and increase the precision of the measurements, while the other two have a larger diameter to increase the rate of triggered events. In addition, there is a $10 \mathrm{~cm}$ thick layer of lead bricks sitting on the top of the bottom scintillator. This 
layer absorbs the soft component of the atmospheric particle showers. Therefore, even though this component can trigger the top and middle scintillators, the atmospheric electrons, or other low momentum non-penetrating particles, will not count as an event since the three-fold coincidence would be missing.

\subsubsection{Data Acquisition System}

Data are recorded with a digital oscilloscope (Tektronix TDS540) linked to a computer by a network connection. To save disk space and to organize data in a more intelligible format, a macro written in $\mathrm{ROOT} \mathrm{C}++$ converts the original data into a ROOT tree. ROOT macros are then used to measure the amplitude, the full width at half maximum (FWHM) of the pulse, and draw the pulses of a dataset.

\subsection{Monte Carlo Simulation}

A Monte Carlo program has been written in order to determine the optical properties of WLS from the experimental results. This program, written under the ROOT $\mathrm{C}++$ framework, converts some input parameters into quantities equivalent to measurements performed with the telescope. The Monte Carlo simulation is rudimentary, but it has the ability to provide an estimate for the number of photons detected by the PMTs for a given detector configuration.

In order to simulate the Čerenkov cone, photons were generated with an opening angle of 41.2 degrees with respect to the direction of propagation of the muon. On the other hand, if the medium in the cell was WLS, the direction of the emitted light was isotropic. Based on the geometry of the cell, the simulation propagated and flagged each photon that hit the PMT. The Monte Carlo 
simulation was used to determine the geometric acceptance of the WLS detector, as it will be discussed in the next section.

\subsection{Geometric Acceptance}

In order to validate the measurements of the WLS telescope, some calculations had to be performed. One essential parameter to calculate is the geometry factor for the percentage of photons being detected by the PMTs. Two methods were pursued: Monte Carlo simulations described in Section 5.2 and analytic calculations. Appendix B shows the details of the analytic calculations. Table 5.1 shows that both the Monte Carlo simulations and the analytic calculations of the geometric acceptance are consistent. In the analytic calculation of the light yield, the muon track was centered on the radial axis of the cylinder. In addition the absorption of water and the wavelength dependence of the number of photons radiated were neglected. For the Monte Carlo simulation, tracks were uniformly distributed over the cell with an zenith angle of zero, which is a good approximation of the expected $\cos ^{2} \theta$ angular distribution for small angles. It was reassuring that restraining the muons in the centre of the cell for the simulation reproduce perfectly the results of the analytic calculations. Since the Monte Carlo simulation with evenly distributed muons is more representative of the reality, its result will be the one retained for the description of the geometric acceptance of the WLS telescope. A crude estimation of the uncertainty on the geometric acceptance factors is calculated from the difference between the two methods used. So, $59.8 \pm 6.8 \%$ of the Čerenkov photons and only $18.9 \pm 1.1 \%$ of the WLS photons are detected by the PMTs. These factors need to be accounted for in an analysis of the increase of the light yield. 


\begin{tabular}{|c||c|c|}
\hline $\begin{array}{c}\text { Type of } \\
\text { light }\end{array}$ & $\begin{array}{c}\text { Analytic } \\
\text { approximation }\end{array}$ & $\begin{array}{c}\text { Monte Carlo } \\
\text { simulation }\end{array}$ \\
\hline \hline Cerenkov & $66.6 \%$ & $59.8 \%$ \\
WLS & $20.0 \%$ & $18.9 \%$ \\
\hline
\end{tabular}

Table 5.1: Geometric acceptance of the WLS telescope for Čerenkov and WLS radiations.

\subsubsection{Improvements}

The critical part of the physics of WLS that is both interesting and necessary still have to be included in the Monte Carlo. The dependence of the absorption coefficients on the wavelength of the photons is capital to measure the amount of light that is shifted. So including the wavelength dependence would reduce the uncertainty on the comparison between Monte Carlo and experimental data.

\subsection{Calibration}

\subsubsection{Single-Photon Calibration}

In order to understand the behavior of the detector, an absolute calibration of the PMTs was performed. With an analog to digital converter (ADC), the amplitude of the single-photon peak was measured. With this technique, no coincidence between the scintillators is needed since only one photo-electron is needed and its origin is unimportant. As expected, a background wall generated by the electronic noise was found, on top of which sits a small peak like the one seen in Figure 5.4. This peak is associated with an amplitude that is proportional to the charge produced by a single photo-electron hitting the first dynode of the PMT. This charge can be associated to a known voltage using a pulse generator. 


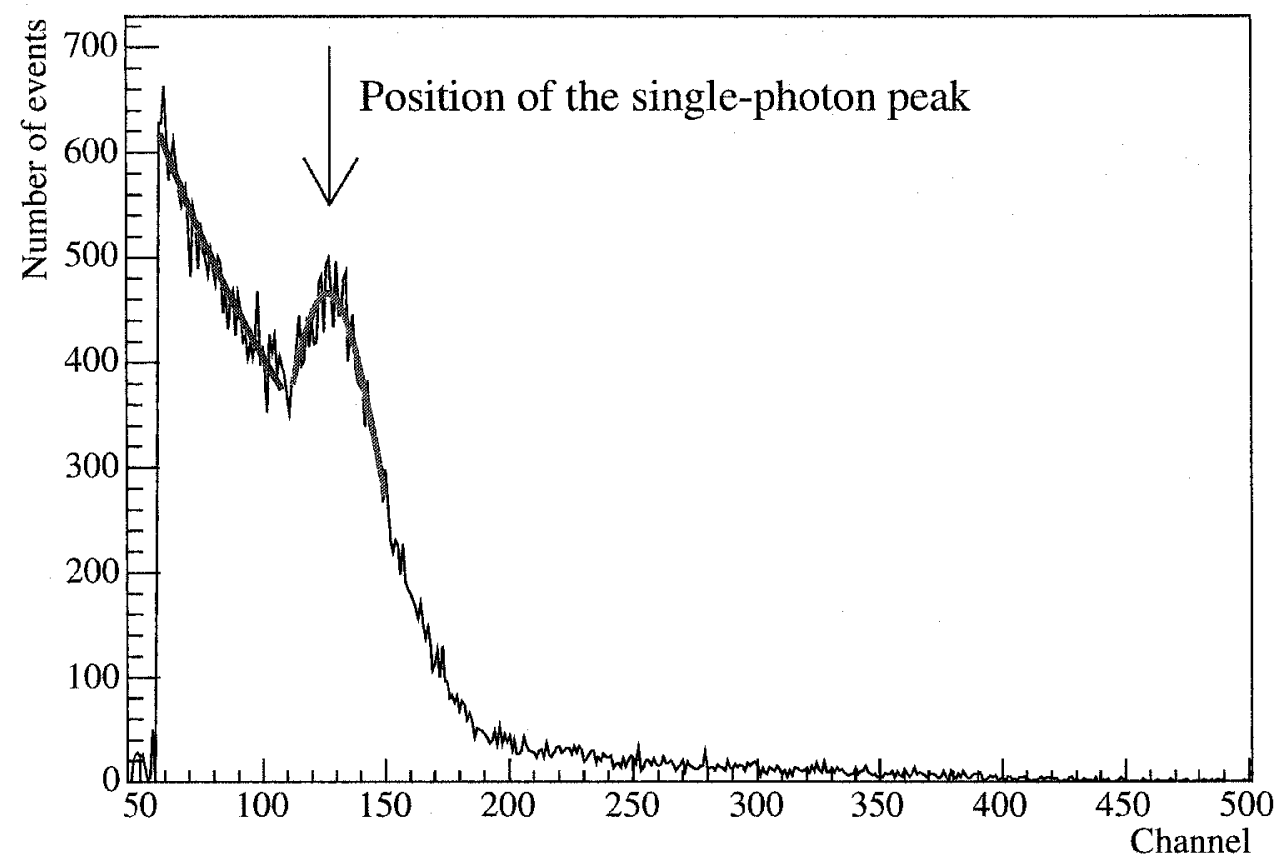

Figure 5.4: Example of a single-photon peak spectrum. The red line represents the electronic noise, while the blue curve represents an excess of events at a voltage corresponding to an electronic cascade initiated by one photo-electron. View this plot in color.

Then, the gain of each PMT was measured, so that the number of photons detected can be determined from the pulse height. Figure 5.5 shows the gain calculated from the position of the single-photon peak as a function of the applied voltage.

\subsubsection{Multi-Photon Calibration}

The second phase of the calibration was performed after adjusting the voltages applied on each of the PMTs so that the amplitudes over many events are 


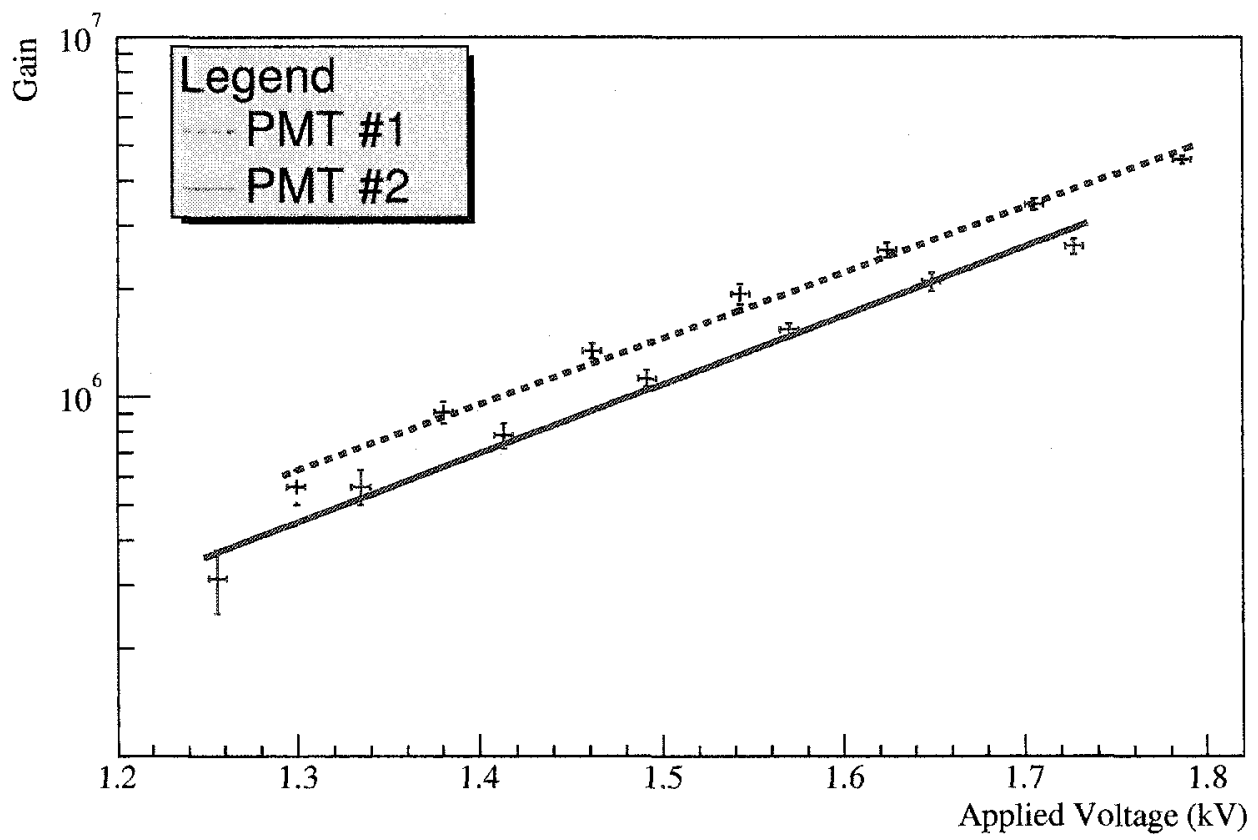

Figure 5.5: Single-photon peak position as a function of the applied voltage on the PMTs. The dashed line corresponds to PMT \#1, while the solid line corresponds to PMT \#2. 
equal for many voltage values (c.f. Figure 5.6). This technique uses the coincidence of the scintillators to choose only photons produced by atmospheric muons. The light deposited in each cell is expected to be the same over a large number of events. This allows the calibration of the gain of one PMT relative to the other. Both calibration techniques indicated that different operating voltages were needed to obtain the same gain for each PMT. Consistent results over many datasets and configurations of the apparatus suggested an optical or physical asymmetry between the gain of the two Čerenkov cells. The results from the multi-photon calibration also suggested that applying voltages differing by 160 volts on the PMTs would compensate for this asymmetry.

\subsection{Timing and Gain Measurements}

The data described in this section were recorded in July 2004 over a period of two weeks. The data consists of ten runs of 741 events each, for two configurations. Both configurations have cell \#1 filled with pure water, while cell \#2 is filled with a Carbostyril solution of $15.4 \mathrm{ppm}$. This concentration was used since the mean free path of photons in a WLS solution needs to be much smaller than the size of the PVC cell. Data were recorded with a voltage of $1900 \mathrm{~V}$ applied on PMT \#1 and $2060 \mathrm{~V}$ on PMT \#2. The orientation of each cell was intermittently changed between runs to test the sensitivity of the experiment to manipulations.

Section 5.5.1 describes the timing calibration of the WLS telescope and Section 5.5.2 summarizes the light gain measurement for WLS molecules. 


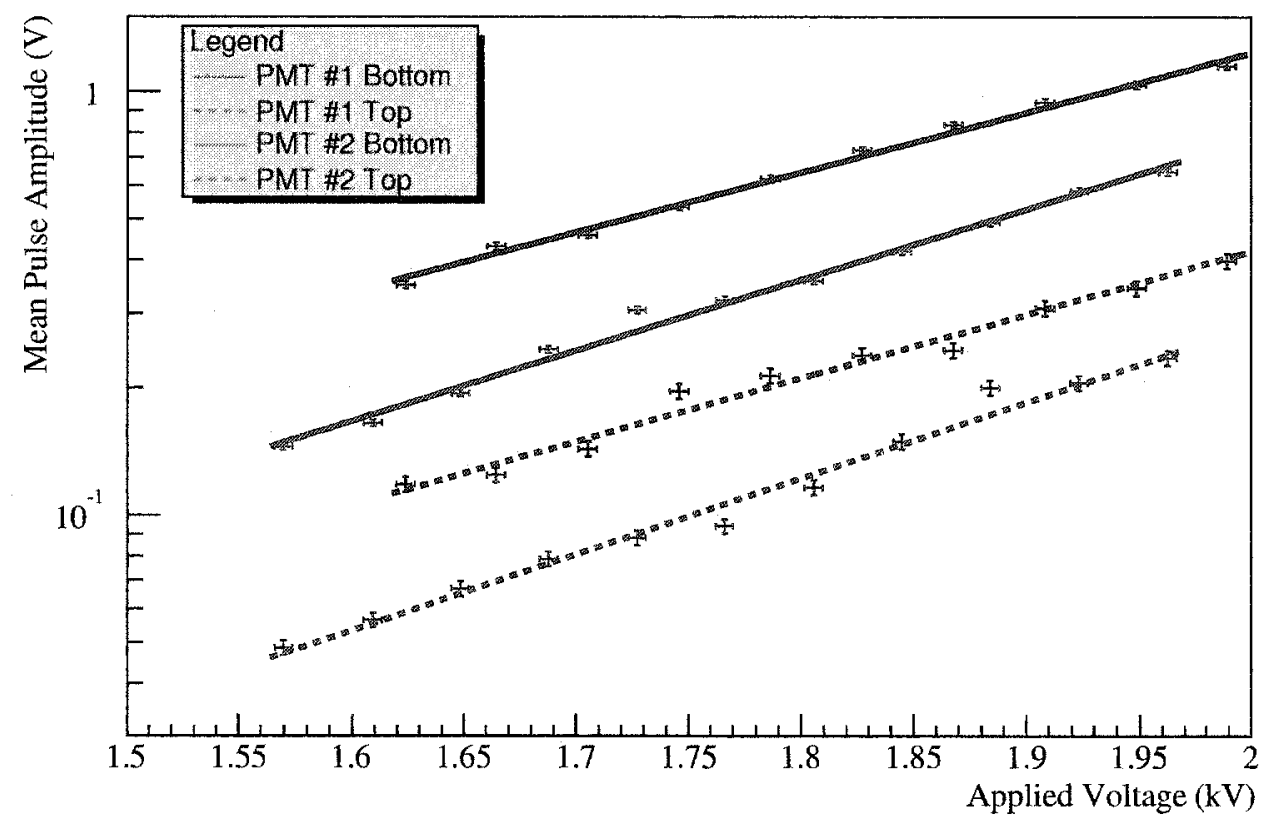

Figure 5.6: Multi-photon mean pulse amplitude as a function of the applied voltage on the PMTs and their orientation with respect to the Cerenkov cone. The dashed lines correspond to the PMTs looking downward, while the solid lines correspond to the same PMTs looking upward. For both configurations, blue corresponds to PMT \#1 and red to PMT \#2. View this plot in color. 


\subsubsection{Time Measurement}

Another property that can be evaluated with the WLS telescope is the fluorescence timing distribution. Section 2.2.5 shows that the measured re-emission delay was approximately 6 ns. Unfortunately, the shortest sampling time of the oscilloscope was only $2 \mathrm{~ns}$, so the resolution of the oscilloscope needs to be compensated by a large dataset. The re-emission time measurements were performed in order to validate the experimental setup of the WLS telescope. Table 5.2 shows the FWHM values of the timing distributions measured from the same dataset used in the previous section.

A naive fit has been performed to measure the decay time of the WLS. Pulse shapes were approximated by Gaussians for both Cerenkov and WLS cells to simulate the spread of the pulse by the electronic components. The simple model used to determine the re-emission time of the isotropic photons is shown in Figure 5.7. The mean of the Gaussian representing the Cerenkov photons was set to a fixed value, while the mean of the WLS peak was variable and represented the mean re-emission time of the isotropic photons. The amplitudes of the Gaussians were taken from Table 5.3, both Gaussian distributions were added and the FWHM of the total distribution was calculated as a function of the mean value of the second Gaussian. The re-emission time at which the FWHM of the model matches the one measured with the "WLS bottom" configuration corresponds to re-emission time measured with the WLS telescope. A value of $6.1 \pm 0.5$ ns was found with this technique, consistent with the $6.17 \pm 0.09 \mathrm{~ns}$ found in Chapter 2. The result associated with the two-Gaussian model for the re-emission time of isotropic photons is presented in Figure 5.8. Based on the good agreement between the timing measurements performed with the WLS 


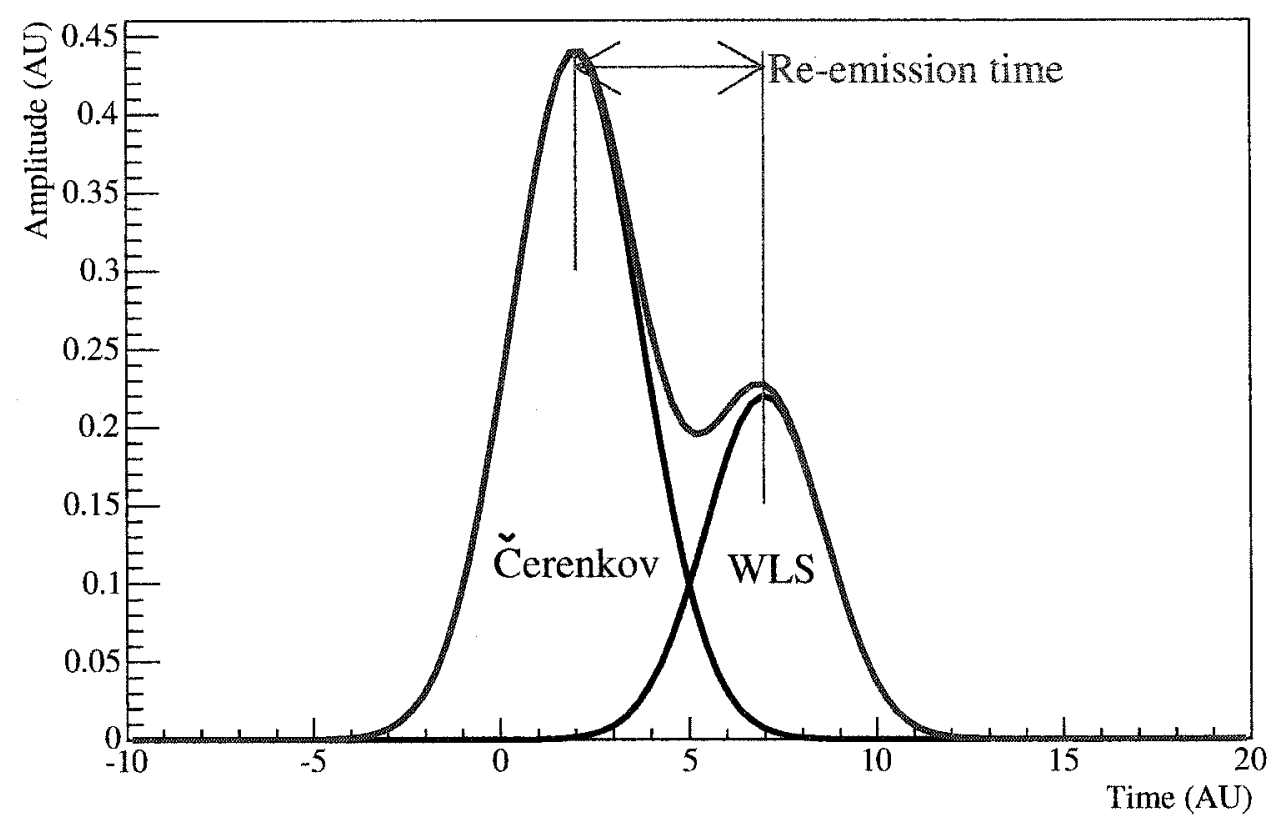

Figure 5.7: Hypothetical situation for the simple model used to determine the re-emission time from pulse widths. Each width and mean amplitude have been measured (c.f. Tables 5.2 and 5.3) so that this model can be used to find the re-emission time. 


\begin{tabular}{|c|c|}
\hline Configuration & $\begin{array}{c}\text { FWHM } \\
\text { (ns) }\end{array}$ \\
\hline \hline WLS top & $12.75 \pm 0.13$ \\
WLS bottom & $13.37 \pm 0.11$ \\
Water bottom & $11.83 \pm 0.13$ \\
\hline
\end{tabular}

Table 5.2: FWHM of the timing distribution for different configurations of the WLS telescope.

telescope and a measurement done with a laser system at Queen's University, the telescope seems to be well calibrated.

\subsubsection{Gain Measurement}

Depending on the orientation of the PMTs, there are two ways to measure the mean amplitude of pulses with and without WLS. If a WLS solution is in the upper cell and water is in the lower cell, only WLS light is detected in the first and Cerenkov light in the second. After inverting the configuration, both Cerenkov and WLS light are measured in the lower cell, while in the upper cell only the dark noise should be detected (c.f. Figure 5.8). Table 5.3 shows the amount of Cerenkov and WLS light measured for each configuration. Since the light in the "WLS bottom" configuration is composed of both WLS and Cerenkov photons, it should equal to the "WLS top" configuration amplitude added to the "pure bottom" configuration amplitude after the subtraction of the dark noise measured in the "pure water top" configuration. Since there is a $12 \%$ difference between the two cases described, the latter value will be considered as a systematic uncertainty on the mean amplitudes.

According to the amplitudes measured from different cell configurations, it 


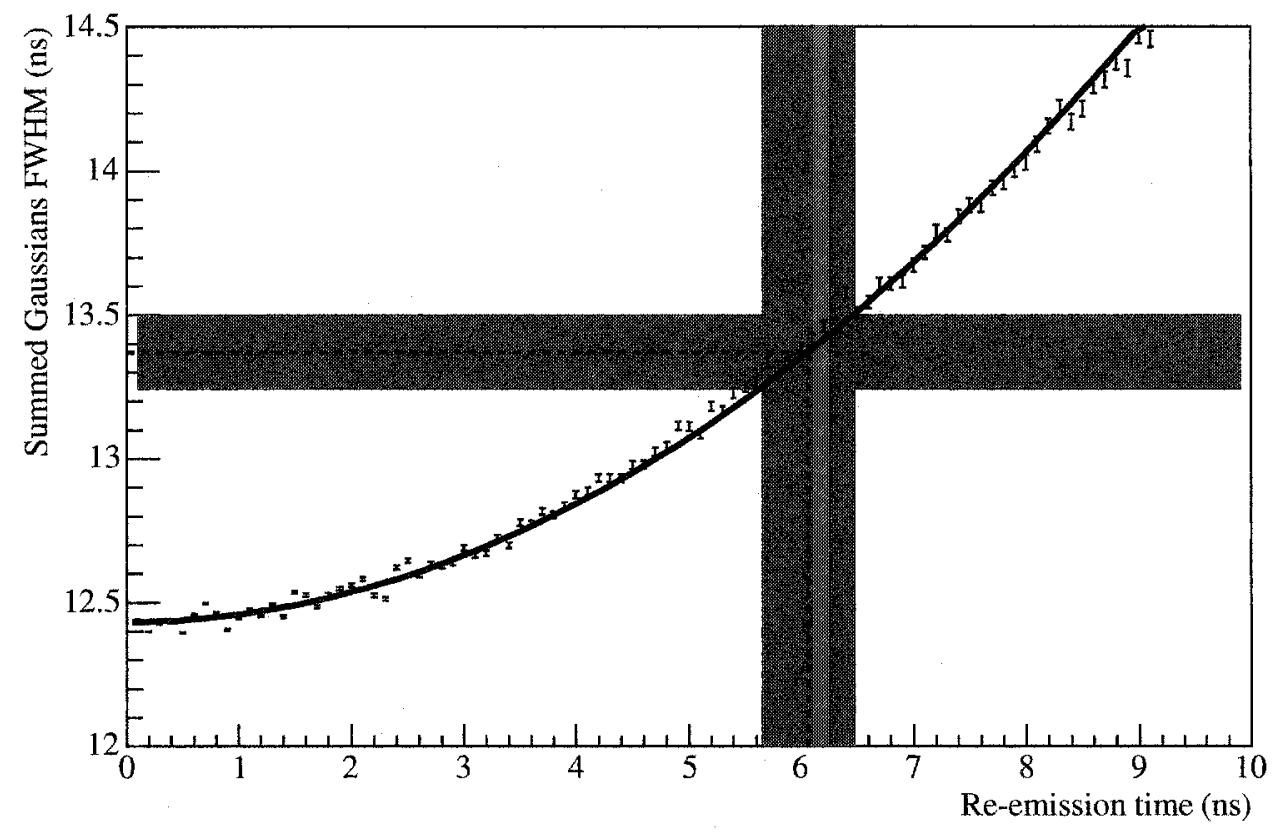

Figure 5.8: The data points and the solid line correspond to the FWHM calculated from the two-Gaussians model as a function of the difference of the mean of the time distributions. The red horizontal band with the associated black dotted line correspond to the measurements of the WLS telescope while the vertical line and band are the corresponding re-emission time according to the model. The blue vertical band corresponds to laser system measurements of the re-emission time. The width of the bands corresponds to the statistical uncertainty. View this plot in color. 
is appropriate to represent the system with those equations:

$$
\begin{gathered}
D_{\breve{C} e r}=G_{\breve{C} e r} \times P_{\breve{C} e r} \\
D_{W L S}=G_{W L S} \times P_{W L S},
\end{gathered}
$$

where $D$ is the number of detected photons, $G$ is the geometric acceptance factor taken from Table 5.1 and $P$ is the number of photons produced inside each cell. The number of detected photons is proportional to the amplitude given in Table 5.3, so the ratio $D_{\breve{C} \text { er }} / D_{W L S}$ is equal to the ratio of the amplitudes after subtracting the dark noise. We can divide the last two equations to obtain:

$$
\frac{D_{\breve{C} e r}}{D_{W L S}}=\frac{G_{\breve{C} e r}}{G_{W L S}} \times \frac{P_{\breve{C} e r}}{P_{W L S}},
$$

which can be rearranged to give

$$
\frac{P_{\breve{C} e r}}{P_{W L S}}=\frac{D_{\breve{C} e r}}{D_{W L S}} \times \frac{G_{W L S}}{G_{\breve{C} e r}} .
$$

Inserting the values for the two fractions on the right-hand side of the equations:

$$
\frac{P_{\breve{C} e r}}{P_{W L S}}=\left[0.97 \pm 0.01_{(a m p)} \pm 0.12_{(i n c)}\right] \times 0.32 \pm 0.06_{(g e o)} .
$$

When the uncertainties, amp from the amplitude from Table 5.3, inc from the $12 \%$ inconsistency described in Section 5.5.2 and geo from the geometry acceptance factor from Section 5.3, are treated properly, the ratio becomes:

$$
\frac{P_{\breve{C} e r}}{P_{W L S}}=0.31 \pm 0.10 .
$$

Finally, the gain in light $\frac{P_{\breve{C} e r}+P_{W L S}}{P_{\breve{C e r}}}$ is found to be $4.2 \pm 1.1$. Note that the main uncertainty is caused by the geometric acceptance factor, which would decrease with a more complete and detailed Monte Carlo simulation. The expected value is 3.6 if the concentration is high enough to shift all short wavelength photons as seen in Section 3.3.1. The measurement is larger than expected, but fully consistent considering the uncertainty. 


\begin{tabular}{|c|c|}
\hline Configuration & $\begin{array}{c}\text { Mean Amplitude } \\
(\mathrm{V})\end{array}$ \\
\hline \hline WLS top & $0.225 \pm 0.004$ \\
WLS bottom & $0.411 \pm 0.008$ \\
Pure water top & $0.075 \pm 0.003$ \\
Pure bottom & $0.220 \pm 0.004$ \\
\hline
\end{tabular}

Table 5.3: Mean amplitude of pulses for different configuration of the WLS telescope.

\subsection{Summary}

The main goal of the WLS telescope has been fulfilled by showing consistent results with the Monte Carlo simulation and with optical property measurements of WLS with spectrometers. Both re-emission time and the light gain showed results consistent with the expected values within their uncertainties. The WLS telescope showed promising results and will be a valuable tool for helping the physics group at Carleton University in the development of the next generation of Čerenkov detectors. 


\section{Chapter 6}

\section{Conclusion}

Wavelength shifter molecules have very interesting optical properties that can enhance water based Čerenkov detectors. The transformation of UV to visible photons significantly increases the number of detected photons per event. Carbostyril 124 and Alexa Fluor 350 are the only two out of five candidates tested that satisfy all the requirements for the SNO experimental set-up. However, Carbostyril has distinct advantages: a narrow absorption spectrum, a large quantum efficiency, and a low net cost.

Monte Carlo studies showed that a $1 \mathrm{ppm}$ concentration of Carbostyril in the heavy water of the SNO detector increases the amount of light detected by a factor of 2.7. A greater number of detected photons per event improves the energy resolution of the detector and reduces the systematic error associated with the energy uncertainty. Increasing the number of detected Cerenkov photons, while keeping the low energy background at the same level, would allow SNO to lower the energy threshold used in the solar neutrino analysis. This would lead to a better sensitivity to spectral distortions and would allow a more precise determination of the neutrino mixing parameters. 
The study confirms that the isotropy of the WLS light could be used to relax the fiducial volume cut and reduce the important uncertainty related to the event vertex position. A more uniform light distribution would also cancel some of the NCD shadowing effect and allow a more symmetrical spatial detector response.

Even if a detector with WLS is more sensitive, a simple test statistic shows that spectral distortions are too small to be seen with reasonable constraints assuming the best SNO fitted point for the mixing parameters; even after reducing the energy threshold down to $3.7 \mathrm{MeV}$ as demonstrated from the analysis which considers the addition of WLS to SNO as a function of the livetime.

An extensive survey of the possibility to incorporate WLS to the SNO detector was pursued. A lot was learned about the properties of Carbostyril 124 and Alexa Fluor 350. As expected, the SNO detector would be more sensitive with WLS. The addition of WLS to SNO would allow tighter constrains on the allowed region of the mixing parameters compare to the present configuration. Unfortunately, it was demonstrated that the addition of WLS to SNO would not lead to the discovery of matter oscillation in several years of operation assuming the preferred mixing solution.

Finally, experimental measurements from the WLS telescope were compared to Monte Carlo studies and consistent results were obtained. Those studies were used for the fine-tuning of the WLS telescope. In the future, more precise measurements from the WLS telescope are expected. This will enable the development of more sensitive detection techniques and therefore allow for more precise determination of elementary particle properties. In conclusion, this thesis has demonstrated that WLS neutrino detectors may have a bright future for the next generation of solar neutrino observatories and long-baseline neutrino factories. 


\section{Bibliography}

[1] SNO Collaboration, Phys. Rev. Lett. 89, 011301 (2002).

[2] H. Chen, Phys. Rev. Lett. 55, 1534 (1985).

[3] S. E. Willis, Ph.D thesis Yale University Sept. 1979, LANL report \# LA8030-T.

[4] G. Badino et al., NIM 185, 587 (1981).

[5] U. Camerini et al., NIM 203, 467 (1982).

[6] X. Dai et al., WLS summary, SNO internal note MANN-5VZPUA, (2004).

[7] L. Wolfenstein, Phys. Rev. D17, 2369 (1978).

[8] S.P. Mikheev and A. Yu. Smirnov, Sov. J. Nucl. Phys. 42, 913 (1985).

[9] SNO Collaboration, Phys. Rev. Lett. 92, 181301 (2004).

[10] X. Shi, D.N. Schramm, and B.D. Fields, Phys. Rev. D48, 2563 (1993). 


\section{Appendix A}

\section{WLS Candidate Structures}<smiles>Cc1cc(=O)[nH]c2cc(N)ccc12</smiles>

Carbostyril 124<smiles>Cc1cc(=O)oc2cc(O)ccc12</smiles>

7-hydroxy-4-methylcoumarin<smiles>Cc1c(C(=O)O)c(=O)oc2cc(O)ccc12</smiles>

7-hydroxycoumarin-3-carboxylic acid<smiles>Cc1c(CC(=O)ON2C(=O)CCC2=O)c(=O)oc2cc(N)c([18OH])cc12</smiles><smiles>Cc1c(CC(=O)O)c(=O)oc2cc(O)ccc12</smiles>

7-hydroxy-4-methylcoumarin-3-acetic acid 


\section{Appendix B}

\section{Analytic Approximation}

\section{B.1 Čerenkov Radiation}

Considering the approximated case of a muon going vertically through the middle of a cell, it is possible to estimate the fraction of the Cerenkov light which is detected by the PMT. The ratio of the propagation length for which the emitted cone hit the PMT $x$, considering the opening angle of the cone, and the radius of the cell, to the height $h$ of the cell is directly related to this fraction. With a $20 \mathrm{~cm}$ diameter cell and a 41.2 degrees opening angle, the value of $x$ is $10 \mathrm{~cm}$. With a total height of $15 \mathrm{~cm}$, the percentage of the track length that produces a detectable photon cone is $66.6 \%$.

\section{B.2 WLS Light}

The analytical approximation for the fraction of WLS light detected by the PMT is performed with a solid angle calculation. This approximation considers an isotropic light source in the center of the cell. With the height $h$ of the cell 
equal to $15 \mathrm{~cm}$ and the diameter $d$ of the cell of $20 \mathrm{~cm}$, the solid angle sustended by the PMT is

$$
\int_{0}^{2 \pi} \int_{0}^{\tan ^{-1} \frac{d}{h}} \sin \phi d \phi d \theta=0.8 \pi
$$

This represents $20 \%$ of the total solid angle. Assuming a point-like and isotropic source, this is also the percentage of the WLS detected by the PMT. 